\title{
Sunjammer: Preliminary End-to-End Mission Design
}

\author{
Jeannette Heiligers ${ }^{1}$ \\ Advanced Space Concepts Laboratory, University of Strathclyde, Glasgow, G1 1XJ, United Kingdom \\ Ben Diedrich $^{2}$ \\ L.Garde, Inc., Tustin, CA, 92780, USA \\ Billy Derbes ${ }^{3}$ \\ BCDAerospace, Healdsburg, CA, 95448, USA \\ and \\ Colin R. McInnes ${ }^{4}$ \\ Advanced Space Concepts Laboratory, University of Strathclyde, Glasgow, G1 1XJ, United Kingdom
}

This paper provides a preliminary end-to-end mission design for NASA's Sunjammer solar sail mission, which is scheduled for ground test deployment in 2015 with launch at a later date and targets the sub- $\mathrm{L}_{1}$ region for advanced solar storm warning. The artificial equilibrium points (AEPs) in the sub- $\mathrm{L}_{1}$ region accessible by the Sunjammer sail as well as solar sail Halo orbits are investigated. Subsequently, the fly-out from an Earth GTO into either a selected sub- $\mathrm{L}_{1}$ AEP or Halo orbit is optimized for a trade-off between the $\Delta V$ to be provided at GTO perigee and the time of flight. In addition, interesting, time-optimal extended mission scenarios are presented to underpin future solar sail mission applications, e.g. transferring to an AEP high above the ecliptic plane for high-latitude Earth observation. All analyses are carried out both for an ideal Sunjammer sail performance as well as for a realistic performance derived from a detailed sail structural analysis. A comparison of the results shows that non-ideal sail properties increase the time of flight of the trajectories by $2.4-7.9 \%$.

\section{Introduction}

SOLAR sailing has long been a theoretical notion, but has recently become reality through JAXA's successful IKAROS mission [1] and NASA's NanoSail-D2 mission [2]. Further solar sail initiatives are scheduled for the

\footnotetext{
${ }^{1}$ Research Associate, Advanced Space Concepts Laboratory, Department of Mechanical and Aerospace Engineering, University of Strathclyde, 75 Montrose Street, Glasgow, G1 1XJ, U.K., jeannette.heiligers@ strath.ac.uk, AIAA member.

${ }^{2}$ Senior Aerospace Engineer, Sunjammer Project, L.Garde, Inc., 15181 Woodlawn Ave, Tustin, CA 92780, USA.

${ }^{3}$ Aerospace Engineer, BCDAerospace, 10520 Wohler Road, Healdsburg, CA, 95448, USA.

${ }^{4}$ Director, Advanced Space Concepts Laboratory, Department of Mechanical and Aerospace Engineering, University of Strathclyde, 75 Montrose Street, Glasgow, G1 1XJ, U.K., colin.mcinnes@strath.ac.uk. 
future, most notably NASA's Sunjammer mission. Scheduled for ground test in 2015 with launch to be determined, Sunjammer is a solar sail Technology Demonstration Mission (TDM) that targets the sub- $\mathrm{L}_{1}$ region (in the SunEarth three-body system) for advanced solar storm warning. By exploiting the continuous acceleration generated by the solar sail, Sunjammer can be stationed closer to the Sun than existing infrastructure at $\mathrm{L}_{1}$ such as SOHO (ESA/NASA, 1996), ACE (NASA, 1997) and WIND (NASA, 2004) as well as future platforms, e.g. DSCOVR (NOAA/NASA, 2015). From such a vantage point, Sunjammer can almost double the warning time for solar storms, which is essential to allow operators of ground and space assets enough time to take appropriate action.

This paper presents a full end-to-end preliminary mission design for Sunjammer. This will include a determination of the artificial equilibrium points (AEPs) accessible by Sunjammer, solar sail Halo orbits around the Sun-Earth line, the optimal fly-out from Earth to the sub- $\mathrm{L}_{1}$ region and novel extended mission concepts. The latter are investigated because at the end of the mission, a unique opportunity arises to demonstrate some of the concepts proposed for solar sails in the literature. These include maintaining artificial equilibria above the ecliptic, e.g. Reference [3], as well as periodic orbits around artificial equilibria displaced away from the ecliptic [4], and transferring between periodic orbits above and below the ecliptic [5]. Therefore, the specific extended mission concepts proposed in this paper include: 1) transfers from a sub- $\mathrm{L}_{1}$ AEP to artificial equilibria high above the ecliptic to observe the Earth's northern high-latitudes; 2) transfers between artificial equilibria above and below the ecliptic to observe both northern and southern hemispheres with one spacecraft; 3) transfers to an AEP trailing the Earth to be ahead of the Earth in the Parker Spiral for a potential further increase in the solar storm warning time and finally, 4) transfers to connect the AEPs associated with the $\mathrm{L}_{1}$ point with those at the $\mathrm{L}_{2}$ point.

Another interesting aspect of this work is a comparison between different solar sail performance models. Preliminary analyses (such as those in Reference [6]) usually consider an ideal solar sail force model, which assumes pure specular reflection of the impinging photons. The solar sail acceleration vector then acts perpendicular to the solar sail surface. While this allows for quick and straightforward analyses, the sail performance will be overestimated. More realistic sail force models exist, such as the optical force model [7] and parametric force model [8]. These take into account non-ideal properties of the sail that generate a sail acceleration component tangential to the sail's surface. Even better, through a detailed structural analysis, realistic Sunjammer sail performance data have been obtained and provided by L'Garde Inc.. This paper therefore conducts the Sunjammer mission analysis both for an ideal Sunjammer sail performance as well as using the real Sunjammer sail data. A comparison between the results of the different sail models will then clearly show the impact of ideal performance assumptions and non-ideal solar sail properties on the mission design.

The paper is structured as follows. First, some background information on the Sunjammer mission is provided in Section II. Section III will subsequently describe the two solar sail models, i.e. the ideal sail model and the real sail model, while Section IV will present the dynamics of the solar sail in the Sun-Earth three-body system. The ideal solar sail force model and dynamics are subsequently used in Section $\mathrm{V}$ to find artificial equilibria and solar sail Halo orbits attainable by Sunjammer. The results from that analysis are used in Sections VI and VII to design optimal fly-out trajectories and optimal extended mission concepts. Finally, the paper ends with the conclusions. 


\section{Sunjammer}

The Sunjammer mission is led by industry manufacturer L'Garde Inc. of Tustin, California, and includes participation by the National Oceanic and Atmospheric Administration (NOAA). Its aim is to demonstrate the propellantless propulsion potential of solar sails and to boost the Technology Readiness Level (TRL) of the L'Garde solar sail from $\sim 6$ to $\sim 9$. It will build on successful ground-deployment experiments led by L'Garde in 2004-2005 and the successful in-space deployment of the NanoSail-D2 mission in 2011. ${ }^{5,6}$ The Sunjammer solar sail was designed to be $38 \times 38 \mathrm{~m}^{2}$ in size. In prior mass estimates, the Sunjammer sailcraft was about $45 \mathrm{~kg}$ and attached to it was a $135 \mathrm{~kg}$ disposable support module. The final configuration of the spacecraft bus is still in development. For the purposes of this analysis, the original design is used. It will be launched as a secondary payload and boost to $\mathrm{L}_{1}$ transfer. Its main objectives are: 1) to demonstrate segmented deployment of a solar sail with $\sim 4$ times the area of that vacuum tested in 2005-2006; 2) to demonstrate attitude control plus passive stability and trim using beam-tip vanes; 3 ) to execute a navigation sequence with mission-capable accuracy; 4) to fly to and maintain position at $\mathrm{L}_{1}$ (e.g. as space weather warning system) and pole-sitter positions.

The Sunjammer mission timeline is presented in Figure 1. The mission will start with launch as a secondary payload on a launch vehicle (e.g. Geosynchronous Transfer Orbit (GTO)). Upon separation, Sunjammer will go to sleep and coast through Earth's radiation belts. Upon waking above the belts, Sunjammer will contact the mission operations center and perform an escape burn to target the initial condition for sailing to sub- $\mathrm{L}_{1}$. The sail will deploy and the carrier with deployment hardware and the escape engine will be jettisoned to save mass. The sailcraft will then execute calibration maneuvers for several days by slewing over a range of angles with observations of the vane angles and trajectory perturbation. This will characterize the moments and forces on the sail at different attitudes to improve attitude control and navigation performance. Following calibration, the Sunjammer sailcraft will sail out to sub- $\mathrm{L}_{1}$ as described below. Before capture into the sub- $\mathrm{L}_{1}$ orbit, Sunjammer will execute recalibration maneuvers to monitor degradation in the sail performance.

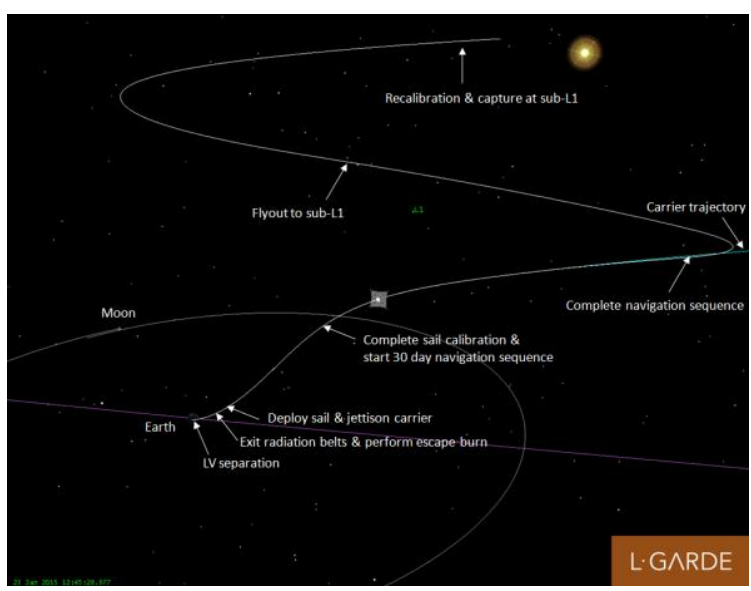

Figure 1 Sunjammer mission timeline.

\footnotetext{
${ }_{6}^{5}$ http://www.nasa.gov/mission_pages/tdm/solarsail/solarsail_overview.html, Retrieved 22 February 2013.

${ }^{6} \mathrm{http} / /$ www.lgarde.com/programs/space-propulsion/sunjammer/, Retrieved 22 February 2013. 3
}

American Institute of Aeronautics and Astronautics 


\section{Sunjammer Performance}

As mentioned in the introduction, this paper considers two different performance models for Sunjammer: an ideal sail model and a real sail model. Details of both models will be provided in the following two subsections.

\section{A. Ideal Sail Model}

The ideal solar sail model assumes that the sail is a perfectly reflecting mirror: the incoming solar photons are specularly reflected and the resulting solar radiation pressure (SRP) force acts perpendicular to the sail surface, i.e. in direction $\hat{\mathbf{n}}$. The SRP acceleration created by the solar sail can then be written as: [7]

$$
\mathbf{a}_{s}=\beta \frac{\mu_{\odot}}{r_{s}^{2}}\left(\hat{\mathbf{r}}_{s} \cdot \hat{\mathbf{n}}\right)^{2} \hat{\mathbf{n}}
$$

with $\mu_{\odot}=1.3272 \times 10^{11}$ the gravitational parameter of the Sun and $r_{s}$ the Sun-sail distance. The parameter $\beta$ is the solar sail lightness number, which is a function of the sail area to spacecraft mass ratio, $\sigma$, and the critical solar sail loading parameter $\sigma^{*}=1.53 \mathrm{~g} / \mathrm{m}^{2}$ :

$$
\beta=\frac{\sigma^{*}}{\sigma}
$$

Equivalently, the lightness number can be defined as the ratio of the solar radiation pressure acceleration and the solar gravitational acceleration. In Section III.B it will be shown that Sunjammer's lightness number equals $\beta=0.0363$.

\section{B. Real Sail Model}

In reality, the sail will perform differently from the ideal case as described in the previous section. For example, due to non-specular properties and wrinkles, the SRP force will not act perpendicular to the sail surface. Instead, a tangential component of the force will exist. Through a detailed structural analysis, L'Garde Inc. have estimated the performance of a realistic sail and expressed this performance through a set of non-dimensionalised force and moment coefficients. The coefficients will be updated using data gathered during calibration maneuvers by the sail after deployment.

These coefficients are defined in the 'Sail' coordinate system, see Figure 2a. The origin of this frame coincides with the sail's center of mass; $\mathrm{z}_{\text {Sail }}$ is directed perpendicular to the sail, $\mathrm{x}_{\text {Sail }}$ points towards the fore beam tip, and $\mathrm{y}_{\text {Sail }}$ completes the right handed coordinate system (and therefore points towards the starboard beam tip). [9]

Clearly, the performance of the sail (i.e. the direction and magnitude of the solar sail acceleration) depends on the attitude of the 'Sail' coordinate system with respect to the Sun. Therefore, a second frame of reference is introduced, which is referred to as the 'Sun' coordinate system, see Figure $2 b$ (note that Figure $2 b$ also includes a third reference frame 'CR3BP' that will be introduced in Section IV). The 'Sun' coordinate system is defined as: origin at the sail's center of mass; $\mathrm{z}_{\text {Sun }}$ points towards the Sun; $\mathrm{x}_{\text {Sun }}$ lies in the ecliptic plane; and $\mathrm{y}_{\text {Sun }}$ completes the right handed coordinate system. [9] 
The attitude of the 'Sail' frame with respect to the 'Sun' frame is defined through three angles: Top $(\tau)$, SunIncidence ('SI' or $\alpha$ ) and FlatSpin ('FS' or $\delta$ ). Starting from the situation where the 'Sail' and 'Sun' coordinate systems coincide (i.e. the sail faces the Sun perpendicularly and the fore-aft-axis is parallel to the ecliptic plane), the rotations required to change the sail's attitude to a desired attitude are:

- First, a rotation around $\mathrm{z}_{\text {Sun }}$ over the Top angle, $\tau$

- Second, a rotation around the newly created y' axis over the SI angle, $\alpha$

- Third, a rotation around the newly created z' axis over the FS angle, $\delta$

Any vector in the 'Sail' coordinate system, $\mathbf{x}_{\text {Sail }}$, can thus be transformed to a vector in the 'Sun' coordinate system, $\mathbf{x}_{\text {Sun }}$, through:

$$
\mathbf{x}_{\text {Sun }}=\mathbf{R}_{z_{\text {Sin }}, \tau} \mathbf{R}_{y ; \alpha} \mathbf{R}_{z ; \delta} \mathbf{x}_{\text {Sail }}
$$

with $\mathbf{R}_{z_{\text {Sun }}, \tau}, \mathbf{R}_{y^{\prime}, \alpha}$ and $\mathbf{R}_{z^{\prime}, \delta}$ the rotation matrices:

$$
\mathbf{R}_{\mathrm{z}_{\mathrm{sin}}, \tau}=\left[\begin{array}{ccc}
\cos \tau & -\sin \tau & 0 \\
\sin \tau & \cos \tau & 0 \\
0 & 0 & 1
\end{array}\right], \mathbf{R}_{y^{\prime}, \alpha}=\left[\begin{array}{ccc}
\cos \alpha & 0 & \sin \alpha \\
0 & 1 & 0 \\
-\sin \alpha & 0 & \cos \alpha
\end{array}\right], \mathbf{R}_{z^{\prime}, \delta}=\left[\begin{array}{ccc}
\cos \delta & -\sin \delta & 0 \\
\sin \delta & \cos \delta & 0 \\
0 & 0 & 1
\end{array}\right]
$$

a)

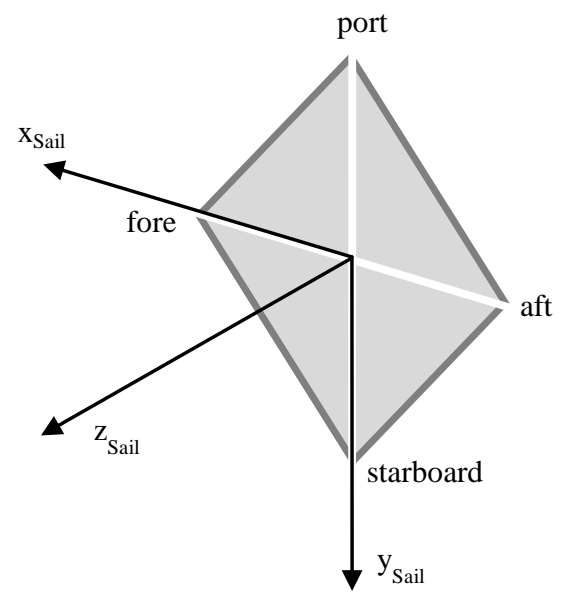

b)

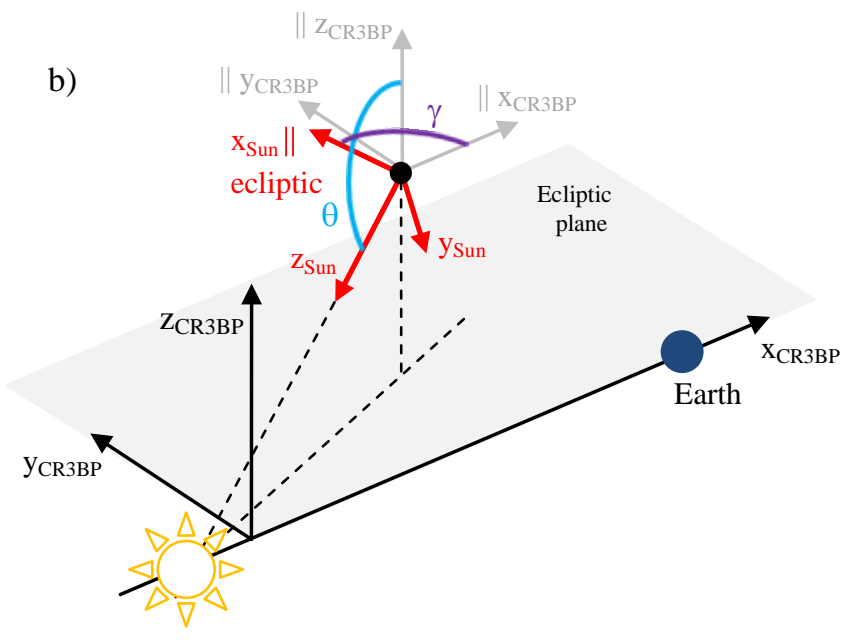

\section{Figure 2 Schematic of a) 'Sail' coordinate system, b) 'Sun' coordinate system.}

Although the attitude of the 'Sail' frame with respect to the 'Sun' frame is defined through 3 angles, the actual performance of the sail (which is defined in the 'Sail' frame) is only determined by the SunIncidence and FlatSpin angles. As stated in Reference [9], the sail may be rotated arbitrarily about the sail-Sun line (i.e. around the $\mathrm{z}_{\text {Sun }}$-axis over the Top angle), without changing forces or moments as expressed in the 'Sail' coordinate system. The resulting data set is therefore a matrix providing the non-dimensionalised force coefficients in the 'Sail' coordinate system for 
a fine mesh in SI and FS angles: the SunIncidence angle runs from -85 to 85 deg with a step size of 1 deg, while the FlatSpin angle runs from -90 to 90 deg with a step size of 5 deg, see the black dots in Figure 3.

Clearly, for $\mathrm{SI}=\mathrm{FS}=0$, the sail produces a force along the $\mathrm{z}_{\text {Sail }}$ axis only (i.e. along the Sun-sail line). Instead, when keeping FS $=0$ and increasing the SunIncidence angle, the force along the Sun-sail line decreases and the force normal to the Sun-sail line increases.

a)

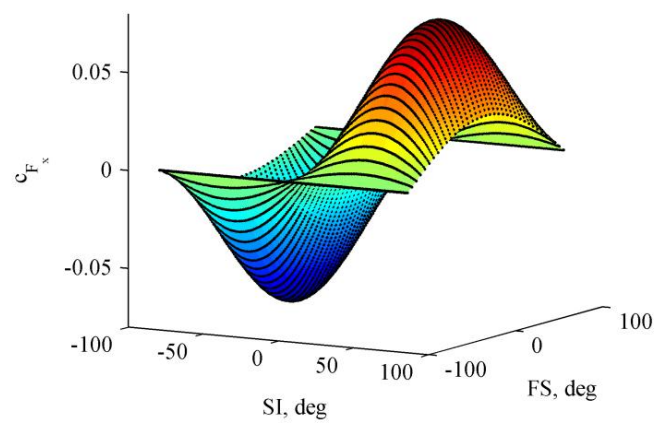

c)

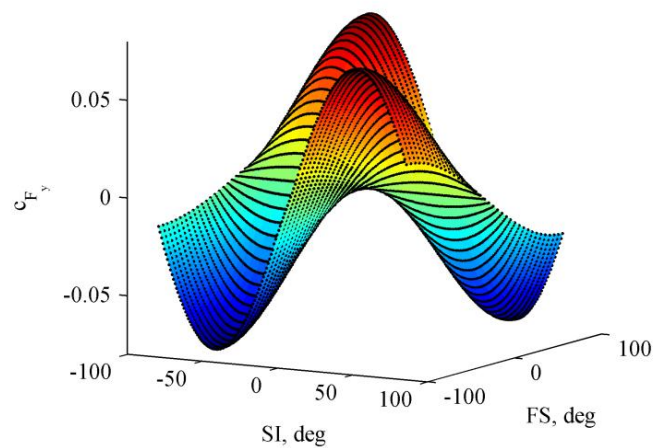

e)

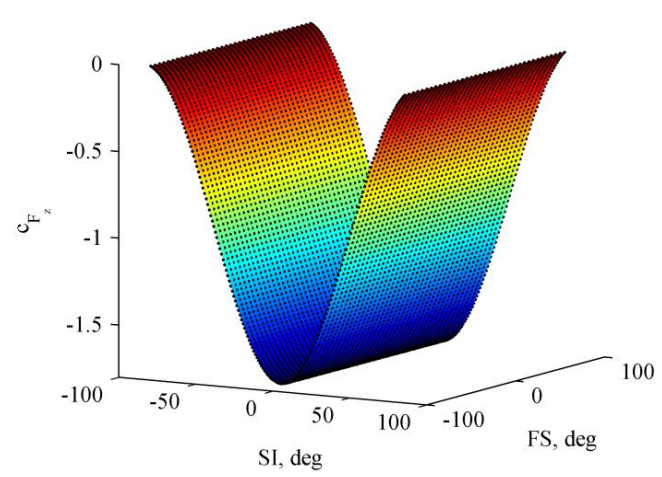

b)

$$
\times 10^{-3}
$$

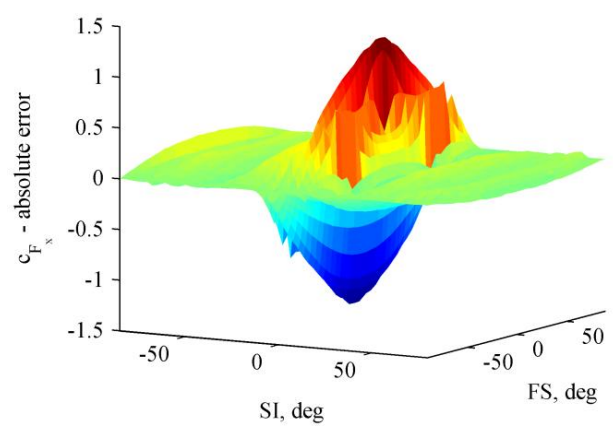

d)

$$
\times 10^{-3}
$$

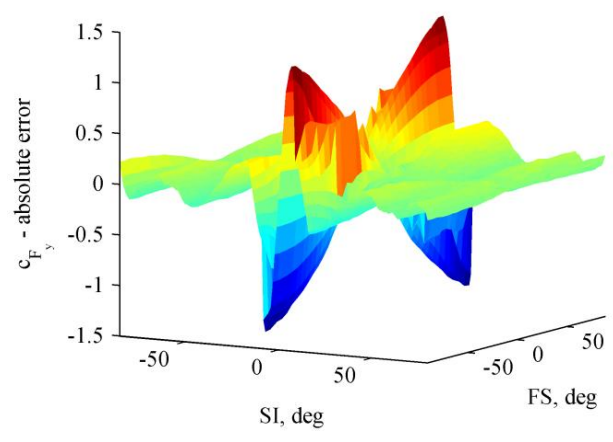

f)

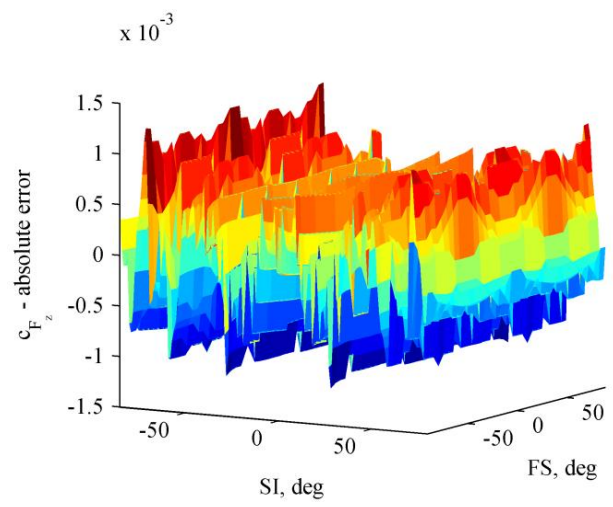

Figure 3 a, c, e) Real Sunjammer data (black dots) and polynomial fit (colored surface) of the nondimensionalised force coefficients. b, d, f) Absolute error of polynomial fit at data points. 
The actual force acting on the sail in $\mathrm{x}_{\text {Sail }}{ }^{-}, \mathrm{y}_{\text {Sail }}$ - and $\mathrm{z}_{\text {Sail }}$-direction can be obtained from the non-dimensionalised force coefficients, $c_{F_{i}}$ with $i=\mathrm{x}_{\text {Sail }}$, $\mathrm{y}_{\text {Sail }}$ or $\mathrm{z}_{\text {Sail }}$, as follows:

$$
F_{i}=c_{F_{i}} P A
$$

with $P$ the local solar radiation pressure and $A$ the nominal sail projected area $\left(1200 \mathrm{~m}^{2}\right)$. Note that the local solar radiation pressure can be calculated as:

$$
P=\frac{W_{E}}{c}\left(\frac{\mathrm{AU}}{d}\right)^{2}
$$

where $W_{E}=1368 \mathrm{~W} / \mathrm{m}^{2}$ is the solar constant at 1 Astronomical Unit (i.e. AU ), $c=299792.458 \mathrm{~km} / \mathrm{s}$ the speed of light in vacuum and $d$ the Sun-sail distance. Clearly, the acceleration produced by the solar sail due to the force in Eq. (5) in $\mathrm{x}_{\text {Sail }}{ }^{-}, \mathrm{y}_{\text {Sail }}{ }^{-}$, and $\mathrm{z}_{\text {Sail }}$-direction can be computed from:

$$
a_{i}=\frac{F_{i}}{m}
$$

with $m=45 \mathrm{~kg}$ the solar sail mass, see Section II.

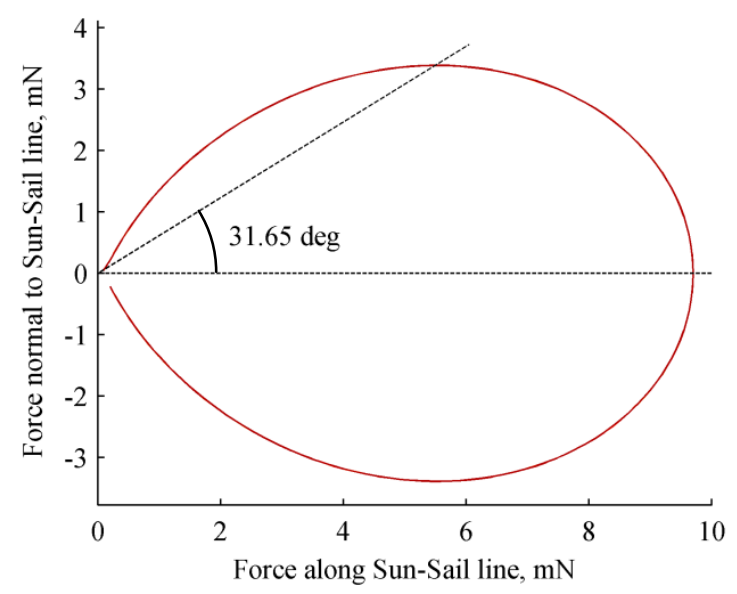

Figure 4 Polar plot of real Sunjammer sail performance at $1 \mathrm{AU}$.

Transforming the performance in Figure 3 into dimensional units results in the polar plot in Figure 4. Again, the figure demonstrates that for SI $=0$, the sail produces a force along the Sun-sail line, while for increasing SI values the force normal to the Sun-sail line increases. The figure furthermore shows that the maximum traverse force is generated for a cone angle (i.e. angle between the normal of the solar sail and the Sun-sail line) of 31.65 deg, which is slightly smaller than for an ideal sail, for which the cone angle to obtain the maximum traverse force is $35.26 \mathrm{deg}$. [7] Finally, a real sail lightness number can be obtained from Figure 4: for a zero-cone angle the acceleration produced by the sail is $0.2155 \mathrm{~mm} / \mathrm{s}^{2}$. Using Eq. (1) this results into a lightness number of $\beta=0.0363$. 
As indicated above, the non-dimensionalised force coefficients are provided for a fine mesh in SunIncidence and FlatSpin angles. In order to obtain Sunjammer's performance for any random combination of SI and FS the data is approximated analytically with a polynomial fit. In fact, two fits are used:

1. First, for each value for the FlatSpin angle, the $c_{F_{i}}(\alpha)$-curves are fitted with a polynomial. This results in 37 $n^{\text {th }}$-order polynomials.

2. Secondly, the $(n+1)$ coefficients of each order term in these 37 polynomials are fitted with an additional $(n+1) n^{\text {th }}$-polynomials.

In mathematical form:

$$
\begin{gathered}
c_{F, i}(\alpha, \delta)=\sum_{j=0}^{n} p_{j}(\delta) \alpha^{j} \\
p_{j}(\delta)=\sum_{k=0}^{n} q_{k} \delta^{k}
\end{gathered}
$$

Both fits are generated using the Matlab ${ }^{\circledR}$ Curve Fitting Toolbox, which allows a maximum order of 9. This corresponds to a total of 300 coefficients: 10 coefficients in Eq. (9) for each of the 10 coefficients in Eq. (8) and multiplied by 3 to separately describe the force coefficient in $\mathrm{x}_{\text {Sail }}{ }^{-}, \mathrm{y}_{\text {Sail }}{ }$, and $\mathrm{z}_{\text {Sail }}$-direction. The values for each of these 300 coefficients are the output of the Curve Fitting Toolbox. Figure 3 shows the result as colored surfaces (in plots a, c, e) as well as the absolute error between the data points (black dots) and the fit (in plots b, d, f). The error is at most one order of magnitude smaller than the absolute value of the force coefficients, but is generally much smaller. Note that, to verify the correctness and accuracy of the polynomial fit to the Sunjammer data set, all results in this paper have also been generated using a simple linear interpolation of the data set. It appeared that very similar trajectories in terms of trajectory and control profile could be obtained for both the fly-out and the extended mission concepts. However, from a computational effort, the polynomial fit performed much better as the sail acceleration can be obtained analytically rather than through an interpolation scheme.

Finally, also note that a 3D-fit in the form

$$
c_{F_{i}}(\alpha, \delta)=\sum_{\substack{j, k=0 \\ j+k \leq n}}^{n} p_{j k} x^{j} y^{k}
$$

would require a significant smaller amount of coefficients (63 instead of 300). However, the accuracy of the fit in terms of absolute error with respect to the actual data appeared to be very poor and significantly overestimated the required time of flights for each of the trajectories considered in this paper.

\section{Solar Sailing in the Three-Body Problem}

The majority of the analyses for the Sunjammer mission are conducted in the well-known Sun-Earth circular restricted three-body problem (CR3BP). In the circular restricted three-body problem (CR3BP), the motion of an infinitely small mass, $m$, (i.e. the solar sail spacecraft), is described under the influence of the gravitational 
attraction of two much larger primary masses, $m_{1}$ (the Sun) and $m_{2}$ (the Earth). The gravitational influence of the small mass on the larger masses is neglected and the larger masses are assumed to move in circular orbits about their common center-of-mass. Figure 5 shows the reference frame that is employed in the CR3BP: the origin coincides with the center-of-mass of the system, the $\mathrm{x}$-axis connects the larger masses and points in the direction of the smaller of the two, $m_{2}$, while the z-axis is directed perpendicular to the plane in which the two larger masses move. The yaxis completes the right handed reference frame. Finally, the frame rotates at constant angular velocity, $\omega$, about the $z$-axis, $\boldsymbol{\omega}=\omega \hat{\mathbf{z}}$. Note that the $\mathrm{x}^{-}, \mathrm{y}-$ and $\mathrm{z}$-axes in Figure 5 correspond to the $\mathrm{x}_{\mathrm{CR}^{3} \mathrm{BP}^{-}}, \mathrm{y}_{\mathrm{CR} 3 \mathrm{BP}^{-}}$and $\mathrm{z}_{\mathrm{CR}^{3} 3 \mathrm{BP}^{-}}-\mathrm{axes}$ in Figure 2b.

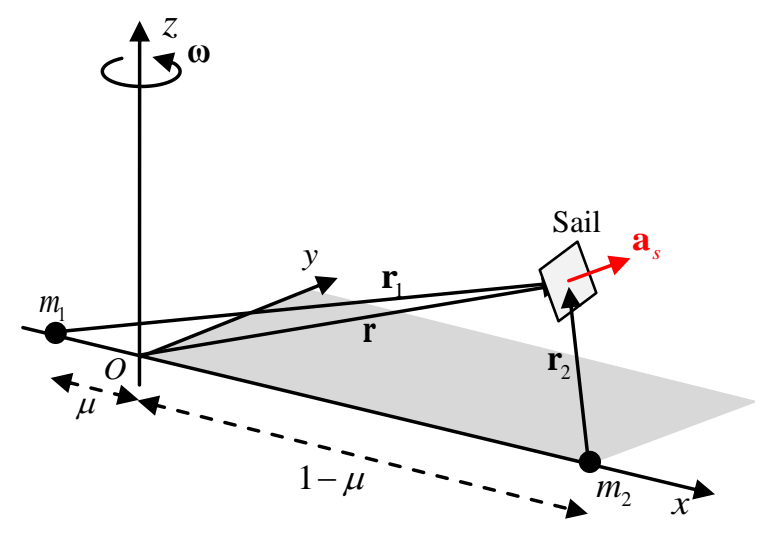

\section{Figure 5 Schematic of circular restricted three-body problem.}

New units are introduced: the sum of the two larger masses is taken as the unit of mass, i.e. $m_{1}+m_{2}=1$. Then, with the mass ratio $\mu=m_{2} /\left(m_{1}+m_{2}\right)$, the masses of the large bodies become $m_{1}=1-\mu$ and $m_{2}=\mu$. As unit of length, the distance between the main bodies is selected, and $1 / \omega$ is chosen as unit of time, yielding $\omega=1$, and so one year is represented by $2 \pi$. In this reference system, the motion of the solar sail is described by:

$$
\ddot{\mathbf{r}}+2 \boldsymbol{\omega} \times \dot{\mathbf{r}}+\boldsymbol{\omega} \times(\boldsymbol{\omega} \times \mathbf{r})=\mathbf{a}_{s}-\nabla V
$$

with $\mathbf{r}=\left[\begin{array}{lll}x & y & z\end{array}\right]^{T}$ the position vector of $m$. The terms on the left hand side are the kinematic, coriolis and centripetal accelerations, respectively, while the terms on the right hand side are the solar sail acceleration and the gravitational acceleration exerted by the primary masses. The gravitational potential, $V$, is given by:

$$
V=-\left(\frac{1-\mu}{r_{1}}+\frac{\mu}{r_{2}}\right)
$$




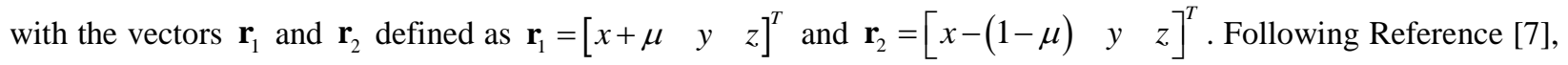
the centripetal acceleration in Eq. (11) can be written as the gradient of a scalar potential function, $\Phi=-\frac{1}{2}\|\boldsymbol{\omega} \times \mathbf{r}\|^{2}$, and can be combined with the gravitational potential into a new, effective potential, $U$ :

$$
U=-\frac{x^{2}+y^{2}}{2}-\left(\frac{1-\mu}{r_{1}}+\frac{\mu}{r_{2}}\right)
$$

The new set of equations of motion then becomes:

$$
\ddot{\mathbf{r}}+2 \boldsymbol{\omega} \times \dot{\mathbf{r}}=\mathbf{a}_{s}-\nabla U
$$

The only term still to be defined is the solar sail acceleration, $\mathbf{a}_{s}$.

\section{A. Ideal sail model}

For an ideal solar sail, the acceleration as given in Eq. (1), can simply be rewritten as:

$$
\mathbf{a}_{s}=\beta \frac{1-\mu}{r_{1}^{2}}\left(\hat{\mathbf{r}}_{1} \cdot \hat{\mathbf{n}}\right)^{2} \hat{\mathbf{n}}
$$

\section{B. Real sail model}

For the real solar sail model, the acceleration can be obtained from the polynomial fit as described in Section III.B. Since that acceleration is given in the 'Sail' coordinate system, two transformations are required: first from the 'Sail' to the 'Sun' coordinate system using Eq. (3) and subsequently from the 'Sun' to the CR3BP coordinate system. A vector in the 'Sun' reference frame, $\mathbf{x}_{\text {Sun }}$, can be transformed to a vector in the CR3BP reference frame, $\mathbf{x}_{\mathrm{CR} 3 \mathrm{BP}}$, using:

$$
\mathbf{x}_{\mathrm{CR} 3 \mathrm{BP}}=\left(\mathbf{R}_{\mathrm{x}_{\mathrm{sin}}, \theta} \mathbf{R}_{z_{\mathrm{Cz} \text { zap }},-\gamma}\right)^{-1} \mathbf{x}_{\mathrm{Sun}}
$$

with $\mathbf{R}_{\mathrm{x}_{\mathrm{sin}}, \theta}$ and $\mathbf{R}_{z_{\mathrm{ckgsa}},-\gamma}$ the rotation matrices:

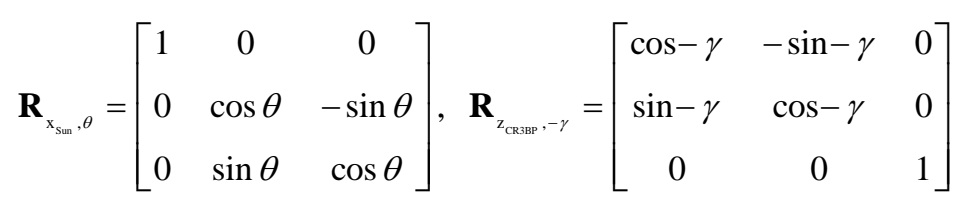

and the angles $\theta$ and $\gamma$ as defined in Figure 2b:

$$
\theta=\tan ^{-1}\left(\frac{z}{\sqrt{(x+\mu)^{2}+y^{2}}}\right)+90^{\circ}, \quad \gamma=\tan ^{-1}\left(\frac{y}{x+\mu}\right)+90^{\circ}
$$

10 


\section{Artificial Equilibrium Points and Solar Sail Halo Orbits}

With the dynamics of the solar sail known in the Sun-Earth three-body system, the artificial equilibrium points (AEPs) and solar sail Halo orbits attainable by Sunjammer can be computed. Note that both are obtained for an ideal solar sail performance only. Maintaining the real Sunjammer sail on these AEPs or Halo orbits would therefore require some form of orbit control, which is considered beyond the scope of this paper but will be considered in future investigations.

\section{A. Artificial Equilibrium Points (AEPs)}

Artificial equilibrium points where the sail can remain stationary within the Sun-Earth three-body system can be derived from Eq. (14) by finding equilibrium solutions, i.e. setting $\ddot{\mathbf{r}}=\dot{\mathbf{r}}=\mathbf{0}$. The required solar sail lightness number to maintain such an AEP can be obtained following the analysis in Reference [7]:

$$
\beta=\frac{r_{1}^{2}}{1-\mu} \frac{\nabla U \cdot \hat{\mathbf{n}}}{\left(\hat{\mathbf{r}}_{1} \cdot \hat{\mathbf{n}}\right)^{2}}
$$

The required sail lightness number is thus only a function of the position within the CR3BP reference frame. Therefore, contours can be drawn in the CR3BP reference frame for constant lightness number. These contours (projected on the $(x, y)$-plane and $(x, z)$-plane) are provided in Figure 6. Note that the grey areas in Figure 6 indicate regions in which no equilibrium solutions exist for the solar sail as these regions would require an acceleration with a component in the direction of the Sun, which the solar sail is unable to generate. Furthermore, by setting Eq. (19) equal to Sunjammer's lightness numbers (see Section III), the equilibrium solutions presented with the white solid line can be obtained. These are the AEPs accessible by Sunjammer.

The yellow cone in Figure 6 indicates the solar exclusion zone: it is well-known that to prevent solar radio interference during communications, the satellite should be located outside the solar exclusion zone when stationed along the Sun-Earth line. In this report, this solar exclusion zone is defined through the Sun-Earth-spacecraft angle, which should have a minimum value of 5 deg [10]. Therefore, rather than being located at the true sub- $\mathrm{L}_{1}$ point (indicated by an open marker in Figure 6), the artificial equilibrium point targeted by Sunjammer in this paper is defined as the AEP in the ecliptic plane, along the $\beta=0.0363$ contour and on the edge of the 5 deg solar exclusion zone. Furthermore, the AEP trailing the Earth is selected, as this will enable a slightly better view on the side of the Sun from which space weather events originate. The coordinates of both the true sub- $\mathrm{L}_{1}$ point and the targeted sub$\mathrm{L}_{1}$ AEP are provided in Table 1.

As Sunjammer will be located a factor 1.6 farther from the Earth than the classical $\mathrm{L}_{1}$ point, it can establish a similar increase in the warning time for space weather events compared to existing infrastructure at $\mathrm{L}_{1}$. 

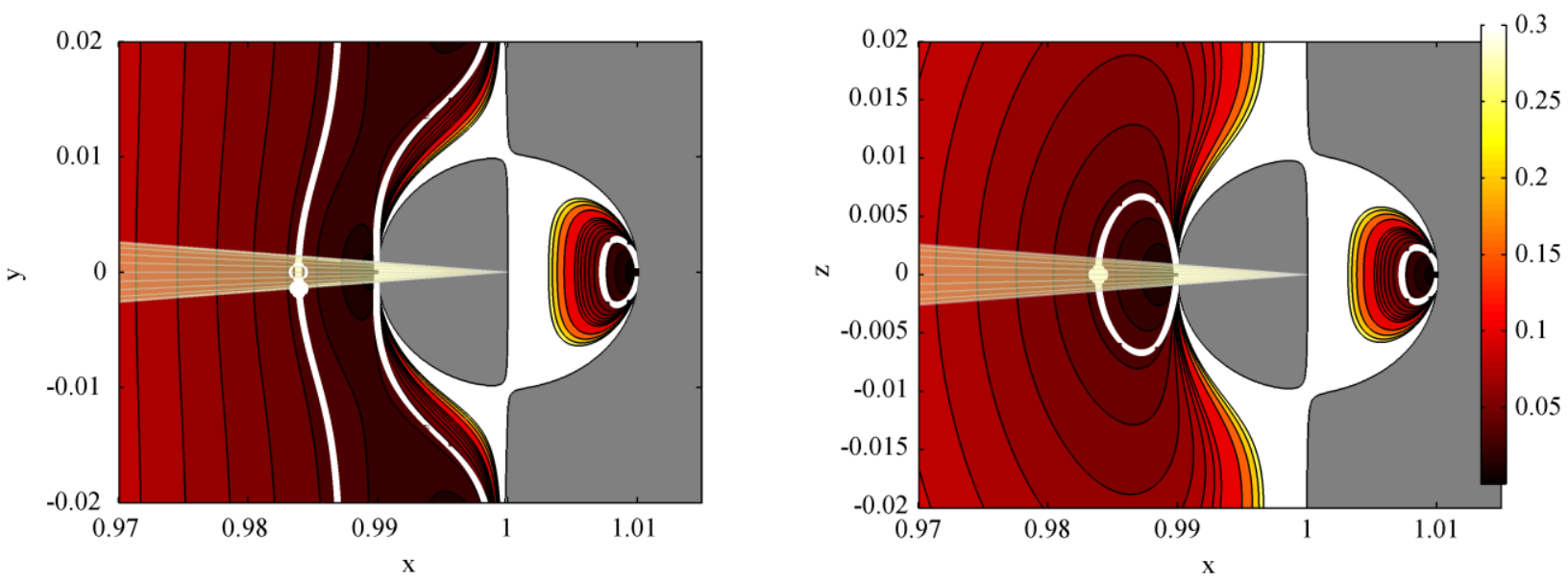

Figure 6 Projected contour plots of constant sail lightness number. The white solid line is the contour for Sunjammer's ideal sail performance of $\beta=\mathbf{0 . 0 3 6 3}$.

Table 1 AEP data for Sunjammer's ideal sail performance of $\beta=0.0363$.

\begin{tabular}{|c|c|c|c|c|c|}
\hline & $x$ & $y$ & $z$ & $\begin{array}{l}\text { Distance from Earth, } \mathrm{km} \\
\text { (measured along the } \mathrm{x} \text {-axis) }\end{array}$ & $\begin{array}{l}\text { Increase in warning } \\
\text { time compared to } \mathrm{L}_{1}\end{array}$ \\
\hline True sub- $\mathrm{L}_{1}$ & 0.983867 & 0 & 0 & $2,412,953$ & 1.611 \\
\hline Targeted sub- $\mathrm{L}_{1}$ AEP & 0.983908 & -0.0144 & 0 & $2,416,471$ & 1.607 \\
\hline
\end{tabular}

\section{B. Solar Sail Halo Orbits}

Rather than being stationary at the targeted sub- $\mathrm{L}_{1}$ AEP, Sunjammer could also be inserted into a solar sail Halo orbit that orbits around the solar exclusion zone. The generation of solar sail Halo orbits around AEPs has been investigated before, see References [11] and [4]. Here, the same approach is adopted, which starts by fixing the attitude of the sail to the one of the true sub- $\mathrm{L}_{1}$ point, i.e. $\mathbf{n}=\left[\begin{array}{lll}1 & 0 & 0\end{array}\right]^{T}$. Furthermore, the equations of motion in Eq. (11) are approximated in the neighborhood of the true sub- $\mathrm{L}_{1}$ point by linearization and expanding the effective potential and solar sail acceleration terms to third order with a Taylor series. Subsequently, the Lindstedt-Poincaré method is used to find the third order solution to this approximated dynamical system. Details on the method can be found in Reference [11].

Since the resulting solar sail Halo orbits are only periodic approximations to the solutions of the full nonlinear system, the orbit quickly diverges when integrating its initial conditions through Eq. (11). A differential correction scheme, see Reference [12], is therefore employed to correct the initial conditions and find true solar sail Halo orbits. 
Finally, by starting with small-amplitude solar sail Halo orbits, a continuation scheme is used to increase the outof-plane amplitude of the orbit up to the point that it fits around the solar exclusion zone. The resulting solar sail displaced Halo orbit is given in Figure 7. Its initial conditions are:

$$
\mathbf{x}_{\text {Halo }, 0}=\left[\begin{array}{llllll}
0.979822 & 0 & 0.001827 & 0 & 0.012830 & 0
\end{array}\right]^{T}
$$
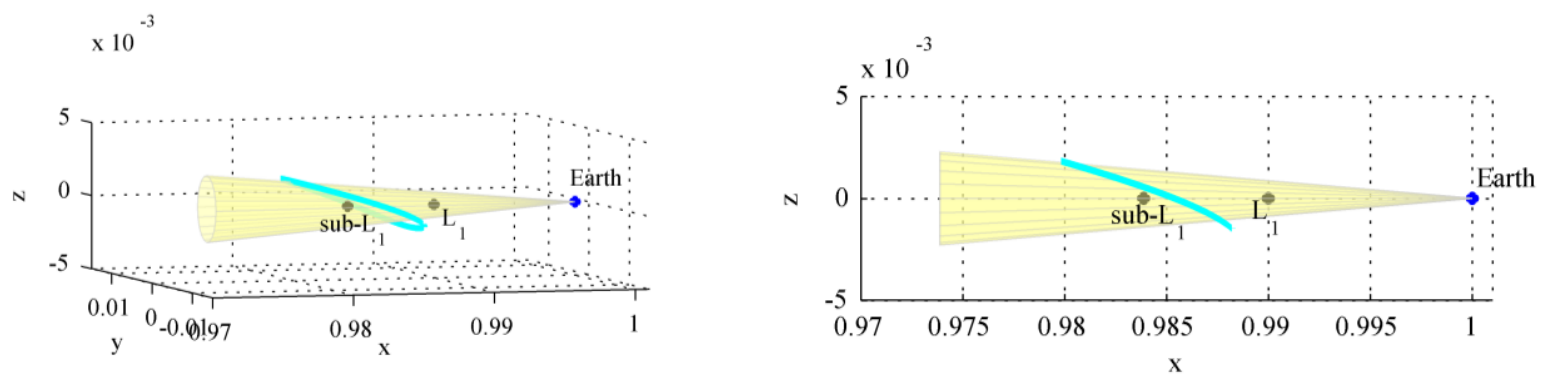

Figure 7 Solar sail Halo orbit for Sunjammer's ideal solar sail performance of $\boldsymbol{\beta}=\mathbf{0 . 0 3 6 3}$.

\section{GTO Fly-Out}

In order to assess the accessibility of the sub- $\mathrm{L}_{1}$ region from Earth, this section finds optimal transfers from GTO to either the targeted sub- $\mathrm{L}_{1}$ AEP (see Table 1) or the solar sail Halo orbit of Figure 7. The section will start with a description of the transfer model, followed by the optimal control problem to be solved and ends with the results.

\section{A. Transfer model}

For the preliminary analysis considered in this paper, it is assumed that the solar sail is launched towards the sub$\mathrm{L}_{1}$ region from a midnight GTO using the upper-stage of a Falcon 9 launcher. This means that perigee of the GTO is located on the night-side of the Earth, i.e. behind the Earth along the Sun-Earth line. Using the inertial reference frame as depicted in Figure 8, the Keplerian elements of the GTO can be defined as in Table 2 (keeping in mind that the time, $t$, is the time during the year measured from winter solstice and in dimensionless form such that 1 year corresponds to $2 \pi$ ).

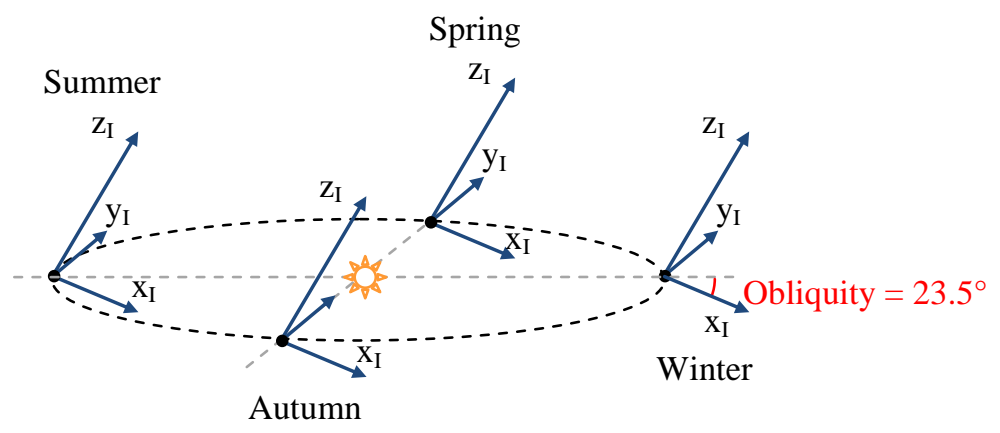

Figure 8 Earth-centered inertial reference frame.

13

American Institute of Aeronautics and Astronautics 
Table 2 Keplerian elements of the Falcon 9 midnight GTO in the reference frame of Figure 8.

\begin{tabular}{ccccc}
\hline$a, \mathrm{~km}$ & $e$ & $i, \mathrm{deg}$ & $\Omega, \mathrm{rad}$ & $\omega, \mathrm{rad}$ \\
\hline 24371 & 0.73009 & 23 & $\frac{3}{2} \pi$ & $\frac{1}{2} \pi+t$ \\
\hline
\end{tabular}

The model further divides the transfer into a near-Earth two-body ballistic phase and an interplanetary CR3BP solar sail phase, see Figure 9.

a)

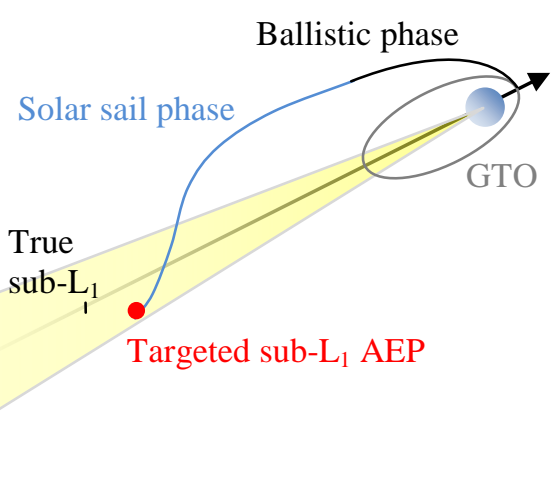

b)

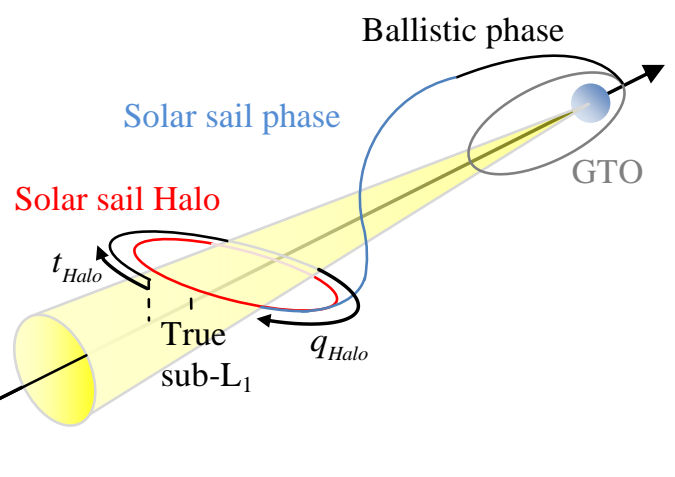

Figure 9 Schematic of solar sail and ballistic phases of transfer from GTO to a) targeted sub- $\mathrm{L}_{1}$ AEP, and b) solar sail Halo orbit.

\section{CR3BP solar sail phase}

The motion of the solar sail during this phase of the transfer is described by the equations of motion given in Eq. (11), i.e. the motion is described in the CR3BP including the use of the solar sail. The end point of this phase should coincide with either the targeted sub- $\mathrm{L}_{1}$ AEP or any point along the solar sail Halo orbit. The initial statevector should be close to the Earth and should be linkable to the GTO through a ballistic two-body arc.

\section{Two-body ballistic phase}

By transforming the initial state-vector of the CR3BP solar sail phase from the CR3BP reference frame of Figure 5 to the inertial frame in Figure 8, the CR3BP solar sail phase is linked to the two-body ballistic phase. This transformation can thus be interpreted as a switch between the three-body and two-body problems at the initial statevector. This transformed initial (now two-body) state-vector corresponds to a bounded orbit around the Earth. The position of the orbit's perigee should coincide with the position of the perigee of the GTO. The velocity at perigee, however, can differ from the velocity at perigee of the GTO and any difference is assumed to be compensated for by the Falcon 9 upper-stage. It is this $\Delta V$ provided at perigee of the GTO, hereafter referred to as $\Delta V_{G T O}$, as well as the time of flight in the solar sail arc that are optimized. Details on the accompanying optimal control problem are given in the next subsection. 


\section{B. Optimal control problem}

The optimal control problem to be solved can be described as follows: finding the state history, $\mathbf{x}(t)$, and control history, $\mathbf{u}(t)$, in the CR3BP three-body phase that minimizes the cost function:

$$
J=\Delta V_{G T O}+\left(t_{f}-t_{0}\right)
$$

which trades-off the required $\Delta V_{\text {GTO }}$ and the time of flight. Since the time of flight is given through $t_{f}$ and $t_{0}$, which represent the dimensionless final and initial time in the solar sail phase, respectively, the terms $\Delta V_{\text {GTO }}$ (in $\mathrm{km} / \mathrm{s}$ ) and $\left(t_{f}-t_{0}\right)$ are of the same order of magnitude. Also note that the optimal control problem only spans the solar sail phase and that the ballistic phase only comes into play when computing $\Delta V_{G T O}$ in the objective function. The ballistic phase is thus not part of the state or control history.

As indicated above, to compute the value for $\Delta V_{G T O}$, the initial state-vector of the solar sail phase is transformed to the inertial reference frame of Figure 8 and is further transformed from Cartesian coordinates to Keplerian elements. These Keplerian elements thus represent the bounded Earth orbit of which the initial state-vector is part. ${ }^{7}$ Then, to find the velocity at perigee of this Keplerian orbit (which in terms of position should coincide with perigee of the GTO), the true anomaly is set to zero and the Keplerian elements are transformed back into Cartesian components. This Cartesian state-vector at perigee is denoted by $\mathbf{x}_{p}=\left[\begin{array}{ll}\mathbf{p}_{p} & \mathbf{v}_{p}\end{array}\right]^{T}$ with $\mathbf{p}_{p}$ the position coordinates and $\mathbf{v}_{p}$ the velocity components.

The time at perigee can also be computed, since the time at the initial state-vector, $t_{0}$, is known and the time in the ballistic arc from perigee up to the initial-state vector, $t_{K}$, can be computed using Kepler's equation. The time at perigee is therefore $\left(t_{0}-t_{K}\right)$, which determines the orientation of the GTO in the inertial frame to ensure a midnight launch. With the orientation of the GTO known, the state-vector at perigee of the GTO, $\mathbf{x}_{\text {GTO }}=\left[\begin{array}{ll}\mathbf{p}_{\text {GTO }} & \mathbf{v}_{\text {GTO }}\end{array}\right]^{T}$, can also be computed with $\mathbf{p}_{\text {GтO }}$ the Cartesian position coordinates and $\mathbf{v}_{\text {GTO }}$ the Cartesian velocity components. From this, the value for $\Delta V_{\text {GTO }}$ can be obtained through:

$$
\Delta V_{\text {GTO }}=\left|\mathbf{v}_{p}-\mathbf{v}_{\text {GTO }}\right|
$$

The state and control vectors, $\mathbf{x}(t)$ and $\mathbf{u}(t)$ are composed of the following state and control variables:

\footnotetext{
${ }^{7}$ Note that if the initial state-vector does not correspond to a bounded Earth orbit (i.e. eccentricity at the start of the solar sail $\operatorname{arc} \geq 1$ ), the eccentricity is artificially transformed through a smooth Heaviside function to be below unity to enable the computation of an objective function value. Another transformation is subsequently applied to penalize this objective function value such that the transfer is discarded in the optimization process. See also Reference [17].
}

15

American Institute of Aeronautics and Astronautics 


$$
\begin{aligned}
& \mathbf{x}=\left[\begin{array}{llllll}
x & y & z & \dot{x} & \dot{y} & \dot{z}
\end{array}\right]^{T} \\
& {\left[\begin{array}{lll}
-1 & -1 & -1
\end{array}\right]^{T} \leq \mathbf{u}=\left[\begin{array}{lll}
n_{x} & n_{y} & n_{z}
\end{array}\right]^{T} \leq\left[\begin{array}{lll}
1 & 1 & 1
\end{array}\right]^{T} \quad \text { for ideal sail model }} \\
& {\left[\begin{array}{lll}
-85 & -90 & 0
\end{array}\right]^{T} \leq \mathbf{u}=\left[\begin{array}{lll}
\alpha & \delta & \tau
\end{array}\right]^{T} \leq\left[\begin{array}{lll}
85 & 90 & 360
\end{array}\right]^{T} \quad \text { for real sail model }}
\end{aligned}
$$

with $x, y, z, \dot{x}, \dot{y}$ and $\dot{z}$ the position and velocity components in the CR3BP reference frame and $n_{x}, n_{y}$ and $n_{z}$ the Cartesian components of the ideal solar sail normal vector in the CR3BP reference frame.

While minimizing the objective function in Eq. (21), the dynamics of the system have to be satisfied (i.e. Eq. (11)) as well as a set of constraints, including event constraints, bounds on the states and time, and path constraints.

Event constraints need to be imposed on both the initial and the final state-vector. Concerning the constraints on the initial-state vector, these should ensure that the position of the perigee of the Keplerian orbit corresponding to the initial-state vector coincides with the position of the GTO perigee:

$$
\mathbf{p}_{p}=\mathbf{p}_{\text {GTO }}
$$

Then, to ensure a midnight launch, constraints on the right ascension of the ascending node and the argument of perigee of this Keplerian orbit should also be imposed (see also Table 2):

$$
\begin{gathered}
\Omega_{i}=\frac{3}{2} \pi \\
\omega_{i}=\frac{1}{2} \pi+\left(t_{0}-t_{K}\right)
\end{gathered}
$$

The constraints on the final state-vector, $\mathbf{x}_{f}$, depend on whether Sunjammer targets the sub- $\mathrm{L}_{1}$ AEP or the solar sail Halo orbit. When targeting the AEP, the final boundary constraints are:

$$
\mathbf{x}_{f}=\mathbf{x}_{A E P}
$$

with the position coordinates of the targeted sub- $\mathrm{L}_{1}$ AEP in Table 1 and the velocity components equal to zero.

When targeting the solar sail Halo orbit, an interpolation scheme is used: a time variable is considered that runs along the Halo orbit, i.e. $0 \leq t_{\text {Halo }} \leq P_{\text {Halo }}$ where $P_{\text {Halo }}$ is the period of the Halo orbit and $t_{\text {Halo }}=0$ coincides with the initial condition as provided in Section V.B, see also Figure 9b. By discretizing the time along the Halo orbit, a large state matrix is created that provides the position and velocity vectors along the Halo orbit at each time step. Finally, an additional static optimisation parameter is added to the optimal control problem, $0 \leq q_{\text {Halo }} \leq P_{\text {Halo }}$, which is used to interpolate the state matrix at $\mathbf{x}_{f}$ and optimizes the location along the Halo orbit where Sunjammer is inserted.

Finally, for the ideal solar sail case, a set of two path constraints have to be considered:

$$
\left.\begin{array}{r}
|\mathbf{n}|=1 \\
\left(\hat{\mathbf{r}}_{1} \cdot \hat{\mathbf{n}}\right) \geq 0
\end{array}\right\} \text { for ideal sail model }
$$

16

American Institute of Aeronautics and Astronautics 
where the first path constraint ensures that the norm of the solar sail normal vector equals unity and the second path constraint prevents the use of a solar sail component in the direction of the Sun, which the solar sail is unable to generate. Note that for the real sail model, the latter is taken into account by suitable bounds on the SunIncidence angle.

The optimal control problem is solved with PSOPT [13], which is a particular implementation of a direct pseudospectral method in $\mathrm{C}++$. In order to initialize the optimization, the initial guess is assumed to be a Hohmann transfer from perigee of the GTO to the sub- $\mathrm{L}_{1}$ region. Clearly, this initial guess has errors mainly due to neglecting third body perturbations from the Sun, but it appeared to provide a stable optimization and quick convergence within PSOPT.

\section{Results}

The results for the optimal fly-out from GTO to either the targeted sub- $\mathrm{L}_{1}$ AEP or the solar sail Halo orbit is provided in Table 2 and Figure 10 to Figure 12. Figure 10 and Figure 11 show the ballistic phase of the trajectory in grey and the solar sail arc in blue (ideal solar sail) or green (real solar sail), where the arrows indicate the direction of the solar sail acceleration. The optimal fly-outs to the targeted sub- $\mathrm{L}_{1}$ AEP take place almost entirely in the ecliptic plane, while the optimal fly-outs to the solar sail Halo orbit contain some out-of-plane motion. Furthermore, plots $\mathrm{c}$ and $\mathrm{f}$ show that the constraint on the midnight launch is satisfied.

Referring to Table 3 and considering that the velocity at perigee of GTO is $10.237 \mathrm{~km} / \mathrm{s}$ (while the local escape velocity is $11.007 \mathrm{~km} / \mathrm{s}$ ), it becomes clear from the values for $\Delta V_{\text {GTO }}$ that the Falcon 9 upper stage has to launch the solar sail almost to escape conditions.

Table 3 Optimized GTO fly-out to the targeted sub- $\mathrm{L}_{1}$ AEP (see Table 1) and solar sail Halo orbit (see Figure 7).

\begin{tabular}{lcccc}
\hline Case & $\Delta V_{\text {GTO }}, \mathrm{m} / \mathrm{s}$ & $\begin{array}{c}\text { Time of flight } \\
\text { ballistic arc, days }\end{array}$ & $\begin{array}{c}\text { Time of flight solar } \\
\text { sail arc, days }\end{array}$ & $\begin{array}{c}\text { Total time of } \\
\text { flight, days }\end{array}$ \\
\hline Ideal sail model & \multicolumn{2}{c}{ To targeted sub-L $\mathrm{L}_{1}$ AEP } & & \\
Real sail model & 746.6 & 9.6 & 181.6 & 191.2 \\
\hline & 747.7 & 20.3 & 186.0 & 206.3 \\
\hline Ideal sail model & \multicolumn{2}{c}{ To solar sail Halo orbit } & & 132.1 \\
Real sail model & 747.1 & 7.0 & 125.1 & 142.7 \\
\hline
\end{tabular}

17

American Institute of Aeronautics and Astronautics 
a)

$$
\text { x } 10^{-3}
$$

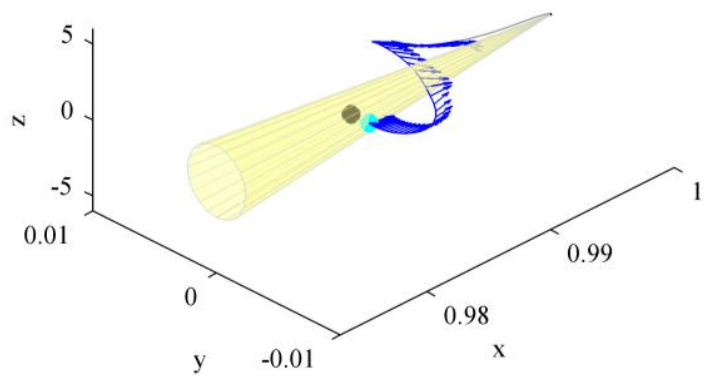

b)

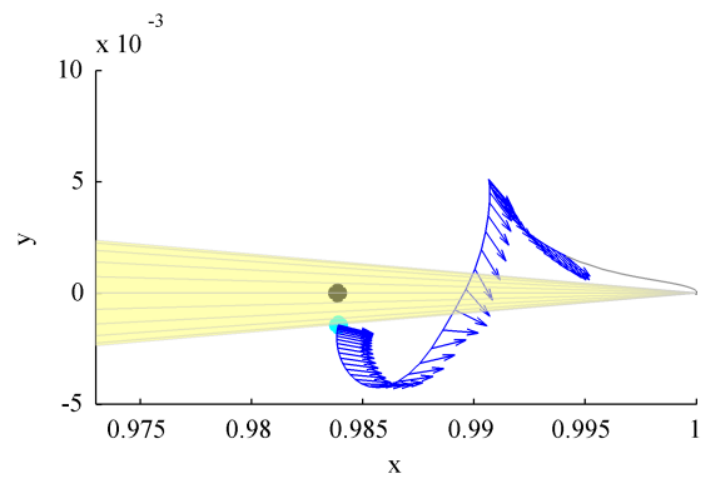

c)

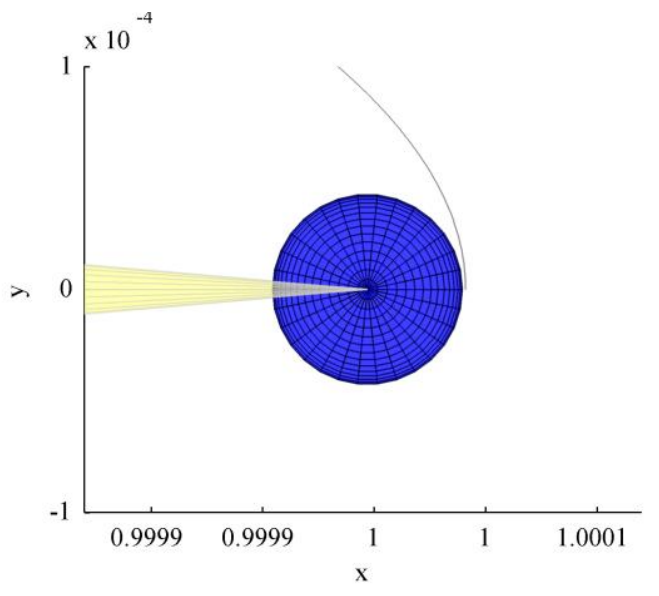

d)

$$
\times 10^{-3}
$$

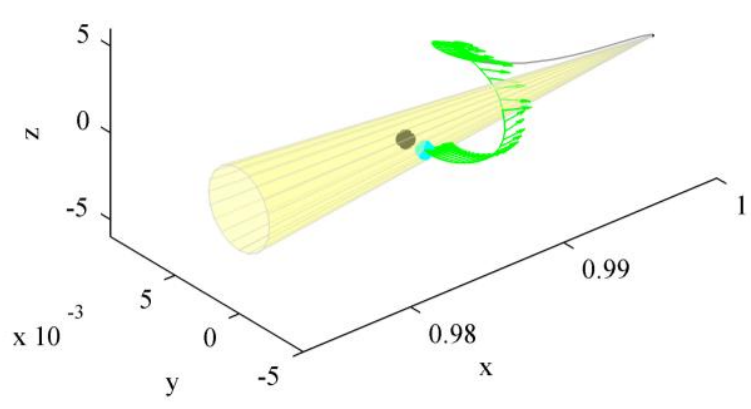

e)

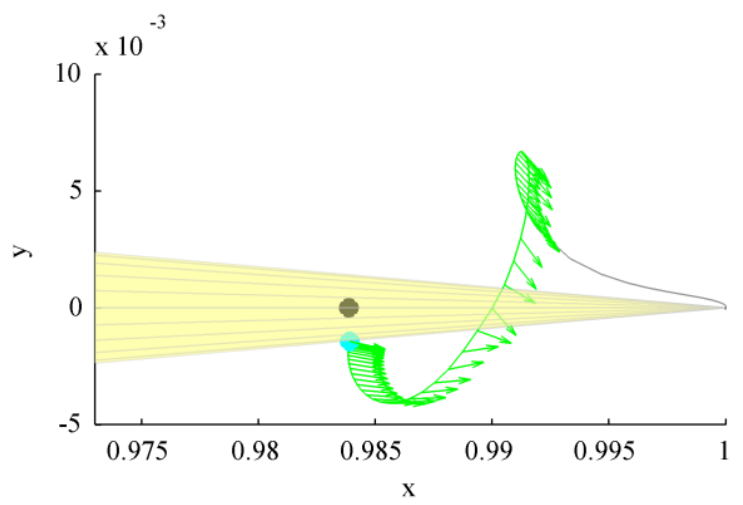

f)

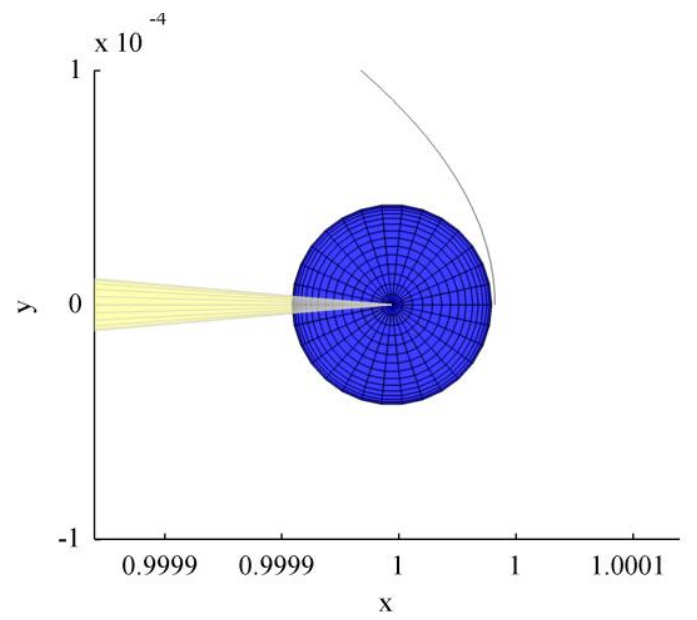

Figure 10 Optimal GTO fly-outs to targeted sub- $\mathrm{L}_{1}$ AEP of Table 1. a-c) Ideal sail performance. d-f) Real sail performance.

18

American Institute of Aeronautics and Astronautics 
a)

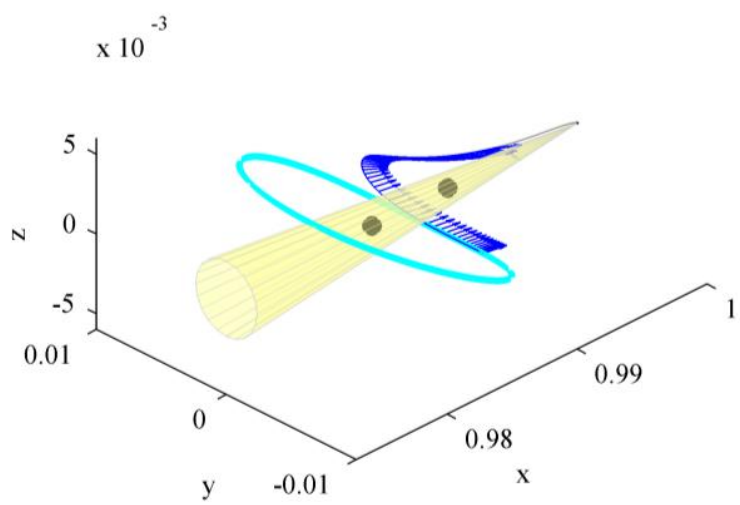

b)

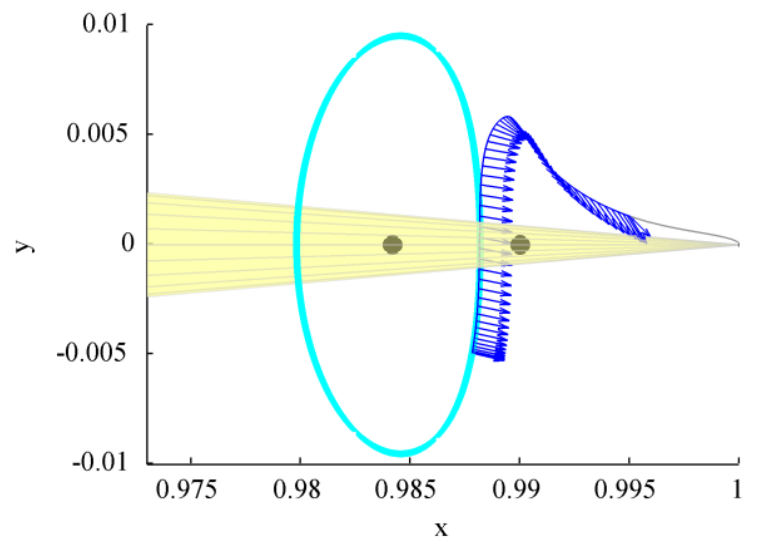

c)

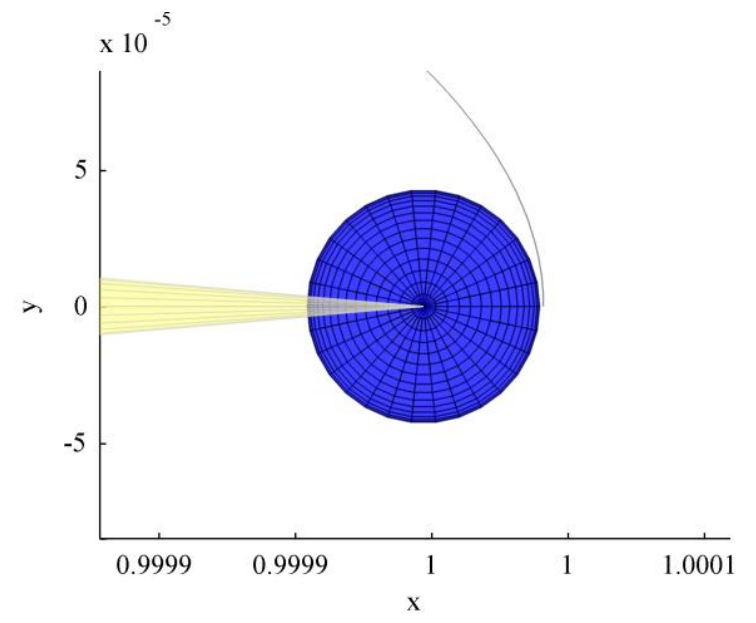

d)

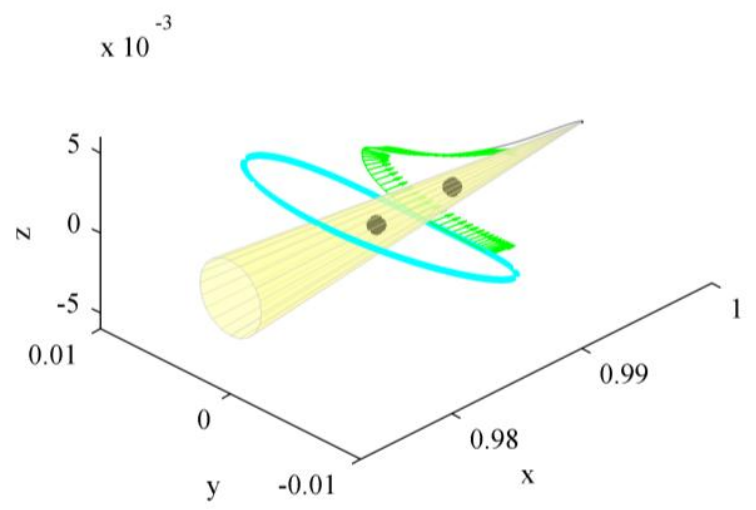

e)

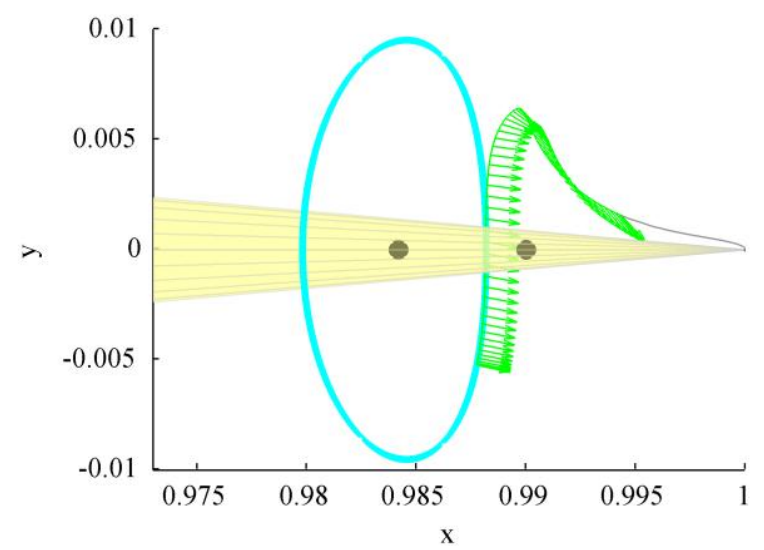

f)

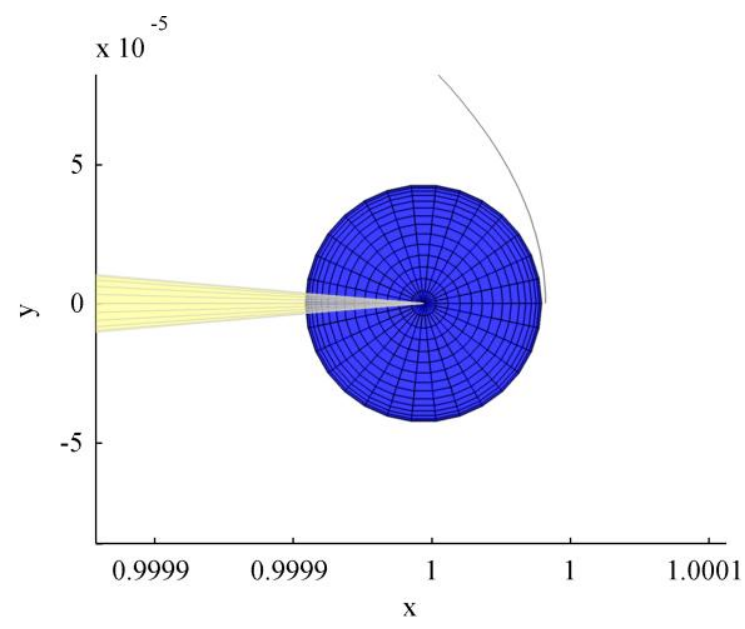

Figure 11 Optimal GTO fly-outs to solar sail Halo orbit of Figure 7. a-c) Ideal sail performance. d-f) Real sail performance.

19

American Institute of Aeronautics and Astronautics 
a)

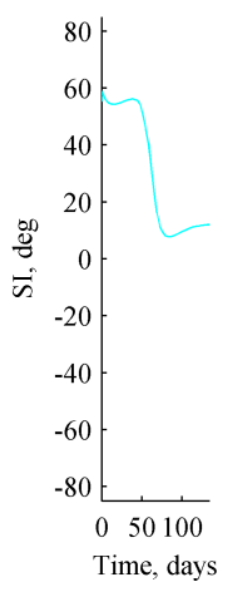

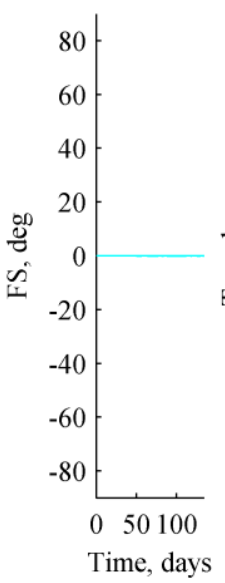

b)

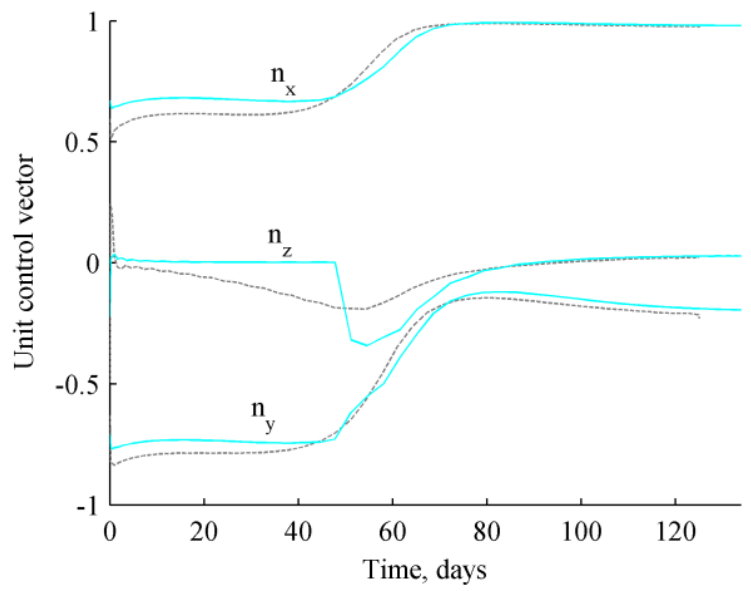

Figure 12 Control profiles of optimal GTO fly-out to solar sail Halo orbit. Blue solid lines are for the real sail performance, dashed grey lines for the ideal sail performance.

Of most interest is the comparison between the ideal and real solar sail performances. Figure 10 to Figure 12 demonstrate that the transfer and control profiles for both solar sail models are very similar: the solar sail is injected into a very high elliptic orbit and starts to lead the Earth in its orbit around the Sun. The sail is then deployed and is used to move back towards the Sun-Earth line to finally arrive at either the sub- $\mathrm{L}_{1}$ AEP or solar sail Halo orbit. The main difference lies in the fact that the trajectory for the real sail performance extends farther from the Sun-Earth line than the trajectory for the ideal sail performance. Furthermore, when looking at the values in Table 3 it becomes clear that the non-ideal properties of the Sunjammer sail do not affect the required $\Delta V_{G T O}$ at GTO perigee to great extent, but do increase the time of flight by $7.9 \%$.

When further examining the control profile of the real sail in Figure 12, it becomes clear that the FlatSpin angle is close to zero, showing its relatively small influence on the solar sail acceleration. The profile for the SunIncidence angle and Top angle show however a more substantial change, which is to be expected as they have the greatest effect on the forces produced by the sail, where the Top angle creates the out-of-plane component of the acceleration.

\section{Extended Mission Concepts}

At the end of the mission, when all mission objectives have been achieved, a unique opportunity arises to use the Sunjammer sail to demonstrate and underpin some of the solar sail concepts and applications proposed in the literature. These concepts include, among others, the use of solar sails to maintain artificial equilibria above the ecliptic for high-latitude Earth observation and communications, see e.g. References [3] and [14]. Transferring the Sunjammer solar sail from the targeted sub- $\mathrm{L}_{1}$ AEP to an out-of-ecliptic AEP could, for example, demonstrate the concept of a pole-sitter spacecraft. Another transfer of interest would be the transfer between AEPs above and below the ecliptic, see for example the work in Reference [5]. This would demonstrate the possibility to perform high- 
latitude observation of both the northern and southern hemispheres of the Earth with one single spacecraft. Inspecting the white contours in Figure 6 that represent AEPs accessible with Sunjammer, the following AEPs can be identified as being of particular interest, see Figure 13:

- North AEP (blue cross): the AEP with the maximum achievable out-of-plane displacement above the ecliptic, which can enable high-latitude telecommunications and Earth observations. [3, 15] The actual out-of-ecliptic displacement that can be achieved from this AEP in terms of spacecraft-Earth-Sun angle is $28^{\circ}$.

- South AEP (green square, coincides with the blue cross in the $(x, y)$-projection in Figure 13a): equivalent to the North AEP but below the ecliptic.

- Parker Spiral AEP (magenta asterisk): the AEP in the ecliptic plane trailing the Earth at an angle of 45 deg. This AEP is ahead of the Earth in the Parker Spiral (more than the 5 deg trailing targeted sub- $\mathrm{L}_{1}$ AEP) and as such can potentially increase the space weather warning time even further. According to Reference [16], due to the rotation of the Sun, fast solar wind streams can catch up with preceding slow solar wind streams. This produces co-rotating interaction regions (CIRs). If the fast solar wind streams dominate over longer periods of time, the interface between fast and slow regimes describes an Archimedean spiral. Under such conditions, a slight increase in the space weather warning time could potentially be achieved if the solar sail would be trailing the Earth.

- $\underline{L}_{2}$-region AEP (yellow diamond): the AEP on the contour associated with the $\mathrm{L}_{2}$-point which will allow for observations on the night-side of the Earth such as geomagnetic tail investigations and astronomical observations.

The coordinates of each of the AEPs of interest are provided in Table 4. The objective now is to connect and establish a tour along each of these AEPs. Starting from the targeted sub- $\mathrm{L}_{1}$ AEP, the AEPs will be visited in the following order: Targeted sub- $\mathrm{L}_{1}$ AEP $\rightarrow$ North AEP $\rightarrow$ South AEP $\rightarrow$ Parker Spiral AEP $\rightarrow \mathrm{L}_{2}$-region AEP.

Table 4 AEP data for extended mission concepts for Sunjammer's ideal sail performance of $\beta=0.0363$.

\begin{tabular}{lccc}
\hline & $x$ & $y$ & $z$ \\
\hline Targeted sub- $\mathrm{L}_{1}$ & 0.983908 & -0.01440 & 0 \\
\hline North AEP & 0.987190 & 0 & 0.006690 \\
\hline South AEP & 0.987190 & 0 & -0.006690 \\
\hline Parker Spiral AEP & 0.986252 & -0.01376 & 0 \\
\hline $\mathrm{L}_{2}$-region AEP & 1.007272 & 0 & 0 \\
\hline
\end{tabular}


a)

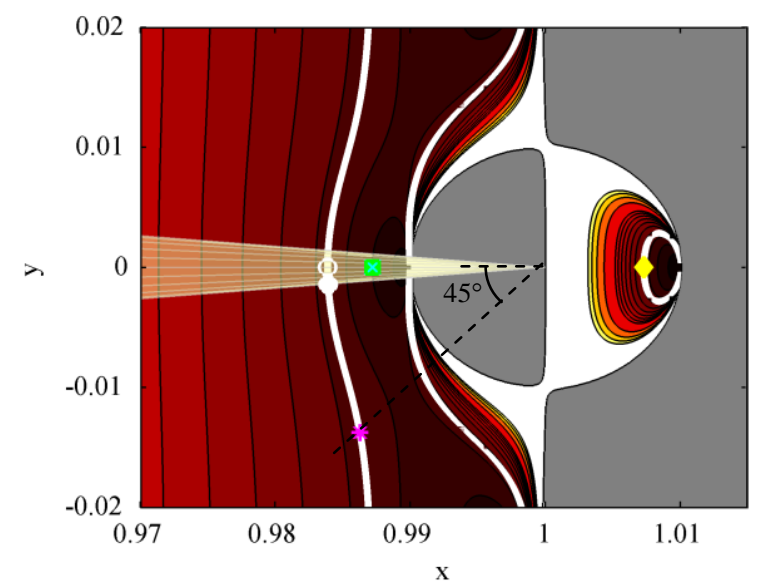

b)

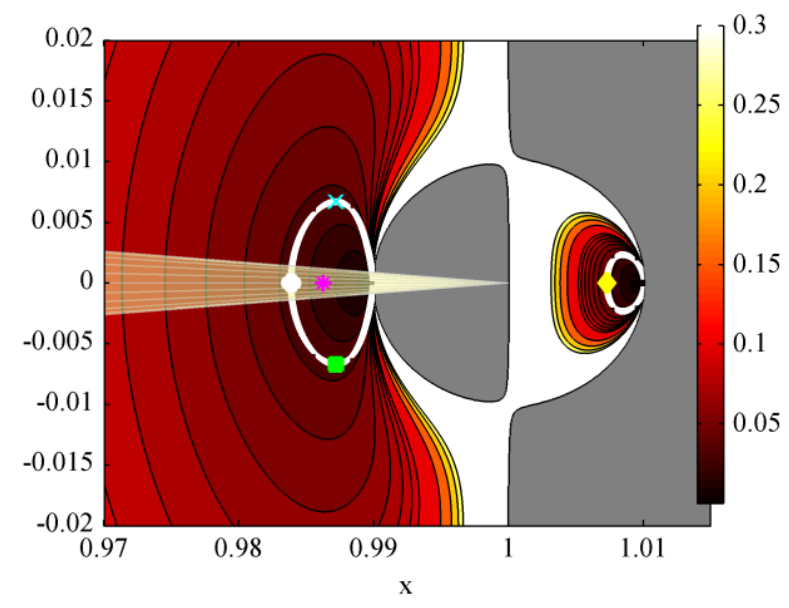

Figure 13 Extended-mission concepts: AEPs of particular interest.

\section{A. Optimal control problem}

The objective now is to minimize the time of flight in each leg of the tour described in the previous section. This requires the solution to a set of optimal control problems. However, the definition of these optimal control problems is very similar to the optimal control problem for the fly-out to the targeted sub- $\mathrm{L}_{1}$ AEP, see Section VI.B: only the objective function and event constraints are different. The objective function now equals the time of flight and the event constraints on the initial and final state vectors, $\mathbf{x}_{0}$ and $\mathbf{x}_{f}$, equal the state vector at the initial and final AEP of the transfer leg under consideration as can be found in Table 4 (with zero velocity components).

Initial guesses for PSOPT are, where possible, chosen to follow the contours of the equilibrium surfaces and the control vector along such an initial guess trajectory is assumed to be the sail normal vector as if each point along the trajectory were an instantaneous AEP. In general, such an initial guess allowed for a smooth optimization process and quick convergence to the optimal solution.

\section{B. Results}

The results in terms of the minimized time of flight for each leg of the tour along the AEPs are summarised in Table 5 while the actual transfers are provided in Figure 14 (blue and grey lines are used to distinguish between the real and ideal sail performances). Again, the arrows indicate the direction of the solar sail acceleration. To limit the amount of results presented, Figure 15 provides the control profiles for only two of the four transfers, i.e. for the sub- $\mathrm{L}_{1}$ AEP to North AEP transfer and the South AEP to Parker Spiral transfer. The controls for the real sail model in Figure 15a and $\mathrm{c}$ are transformed into Cartesian components to provide a direct comparison with the ideal sail model controls in Figure 15b and d. 
Some particular observations are:

- Almost all transfers are smooth both in transfer and control profiles and for some transfers (e.g. the North to South transfer) only very limited steering effort is required from the sail. Other transfers, such as the Parker Spiral AEP to $\mathrm{L}_{2}$-region AEP require a more demanding trajectory and steering profile.

- When comparing the transfer and control profiles for the ideal and real sail models, it can be concluded that both produce very similar results. However, as expected, the non-ideal properties of the real Sunjammer sail introduce a penalty on the time of flight of $2.4-7.3 \%$. The increase in the total time of flight (i.e. of the entire tour) is $5.5 \%$.

- The North AEP to South AEP transfer requires a transfer time of approximately a quarter of a year, which allows observing the northern and southern hemispheres of the Earth during their respective summers of the same year.

- Finally, the control profiles in Figure 15a and c confirm the observation made for the fly-out trajectories in Section VI.C that the FlatSpin angle has a very limited effect on the solar sail acceleration as it is again close to zero.

Table 5 Optimized time of flights in days for the extended mission concepts.

\begin{tabular}{lccccc}
\hline Case & $\begin{array}{c}\text { Targeted sub-L } \\
\text { to North AEP }\end{array}$ & $\begin{array}{c}\text { North AEP to } \\
\text { South AEP }\end{array}$ & $\begin{array}{c}\text { South AEP to } \\
\text { Parker Spiral } \\
\text { AEP }\end{array}$ & $\begin{array}{c}\text { Parker Spiral } \\
\text { AEP to L } 2^{-} \\
\text {region AEP }\end{array}$ & Total \\
\hline Ideal sail model & 109 & 84 & 233 & 252 & 678 \\
Real sail model & 113 & 86 & 250 & 266 & 715 \\
\hline $\begin{array}{l}\text { Percentage } \\
\text { increase }\end{array}$ & 3.5 & 2.4 & 7.3 & 5.6 & 5.5 \\
\hline
\end{tabular}

a)
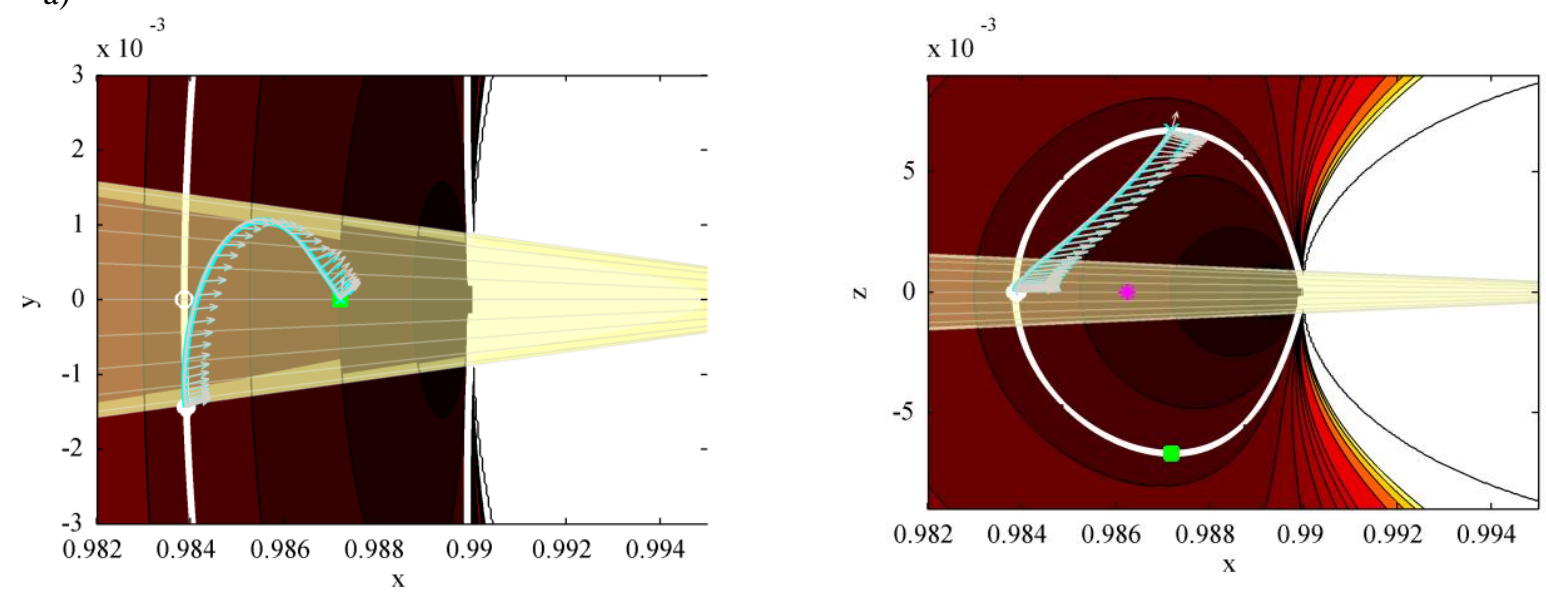
b)
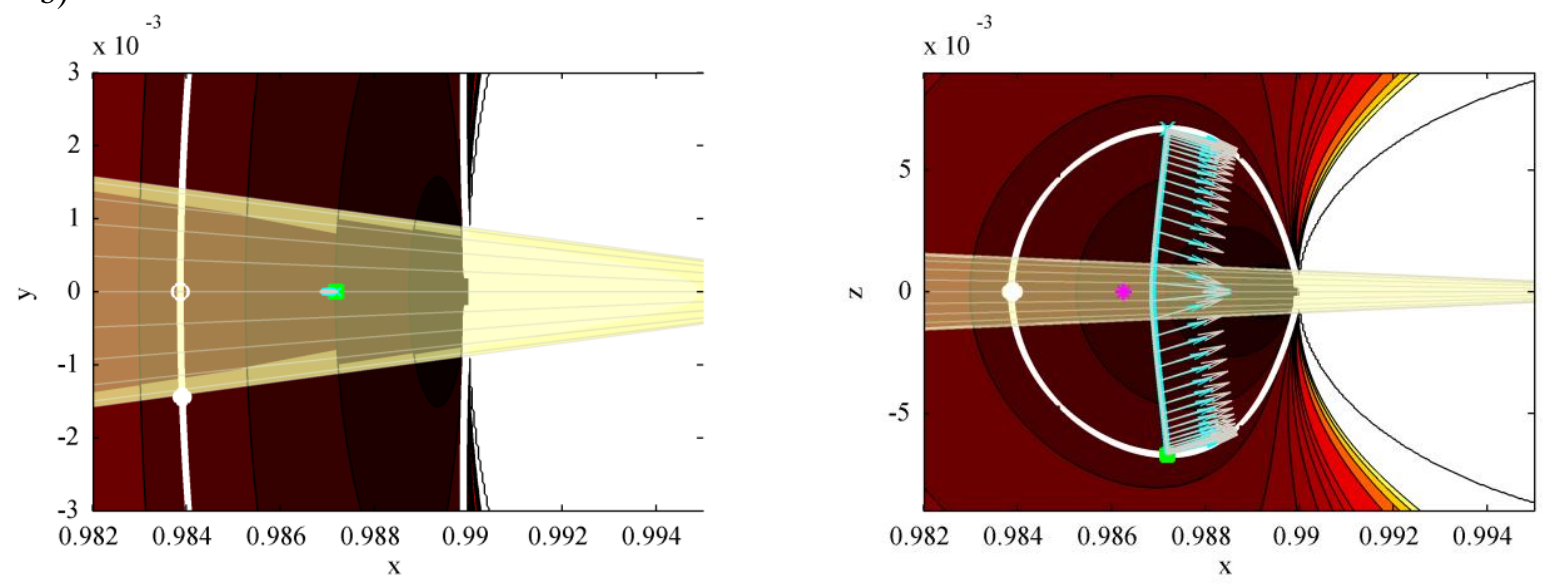

c)
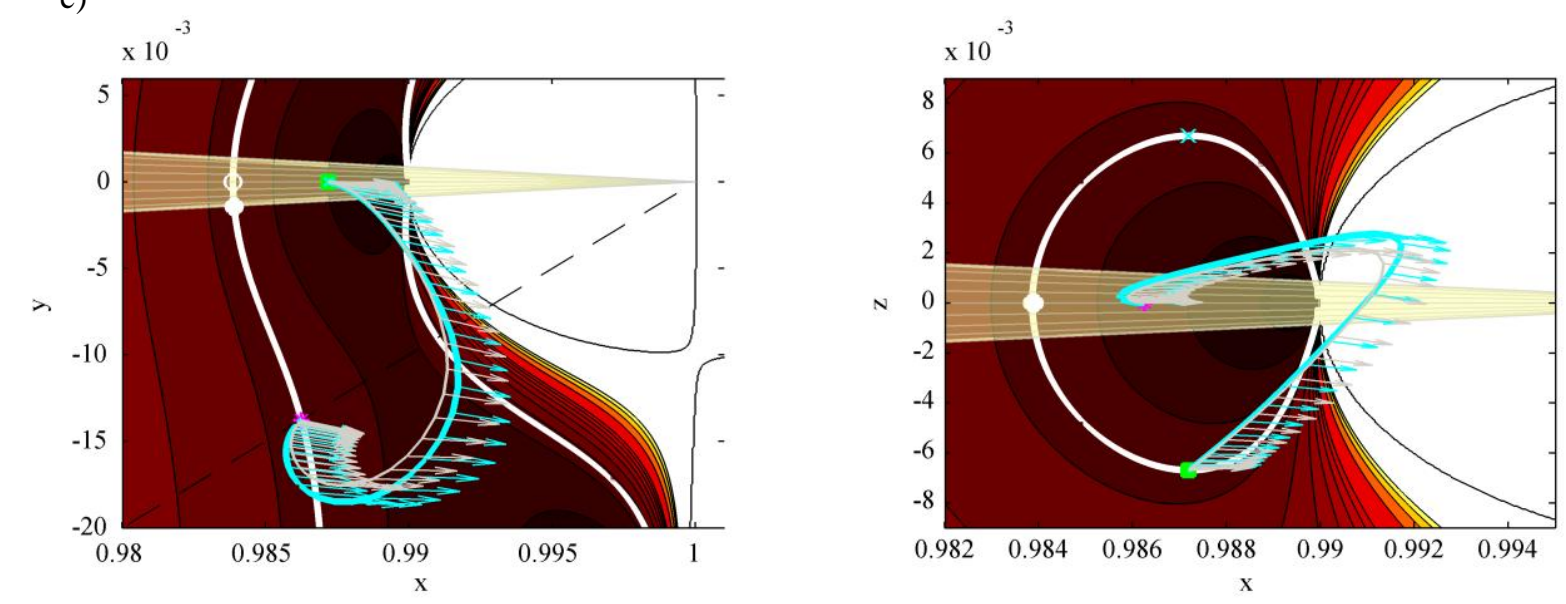

d)
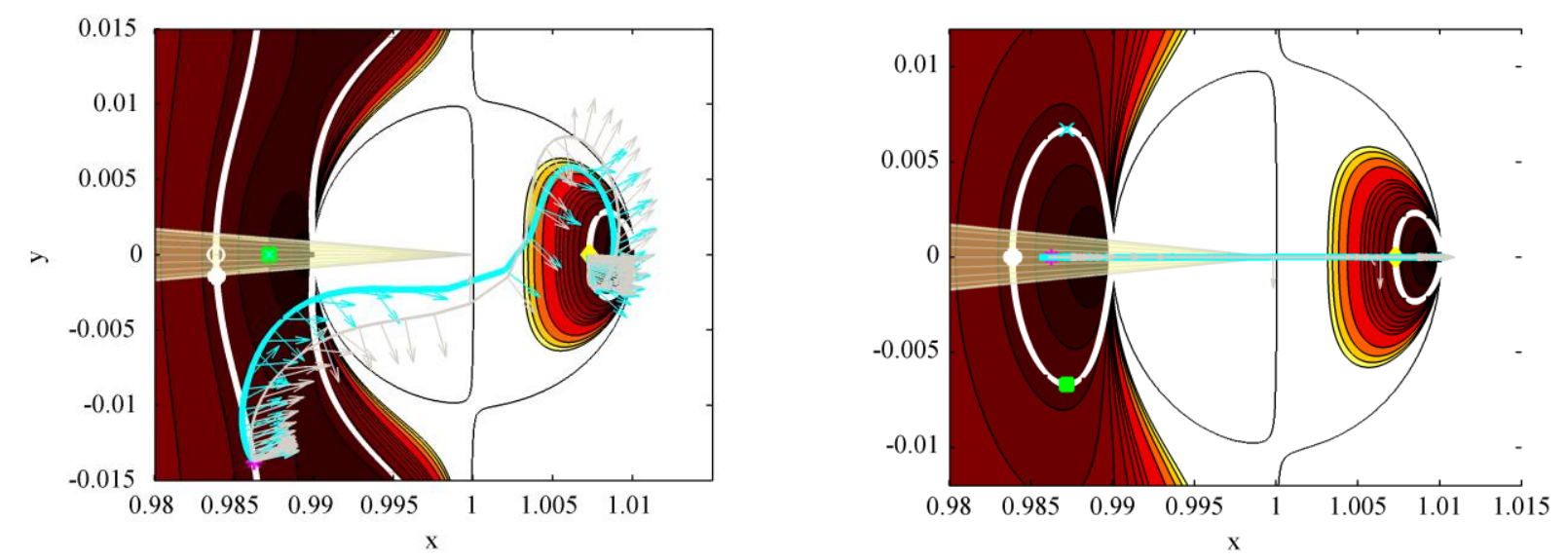

Figure 14 Time-optimal extended mission concepts. Arrows indicate the direction of the solar sail acceleration. Grey and blue lines represent the ideal and real sail models, respectively. a) Targeted sub- $\mathrm{L}_{1}$ AEP to North AEP. b) North AEP to South AEP. c) South AEP to Parker Spiral AEP. d) Parker Spiral AEP to $L_{2}$-region AEP. 
a)

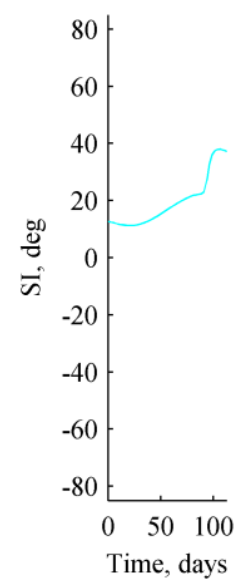

c)

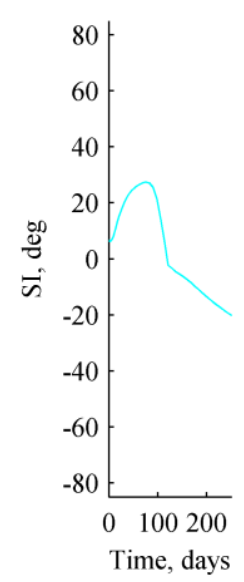

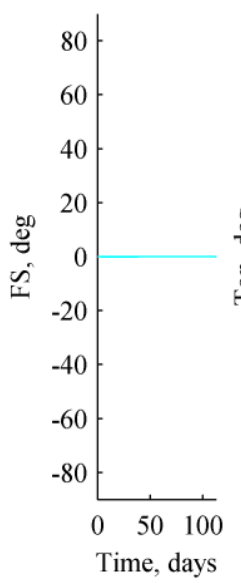
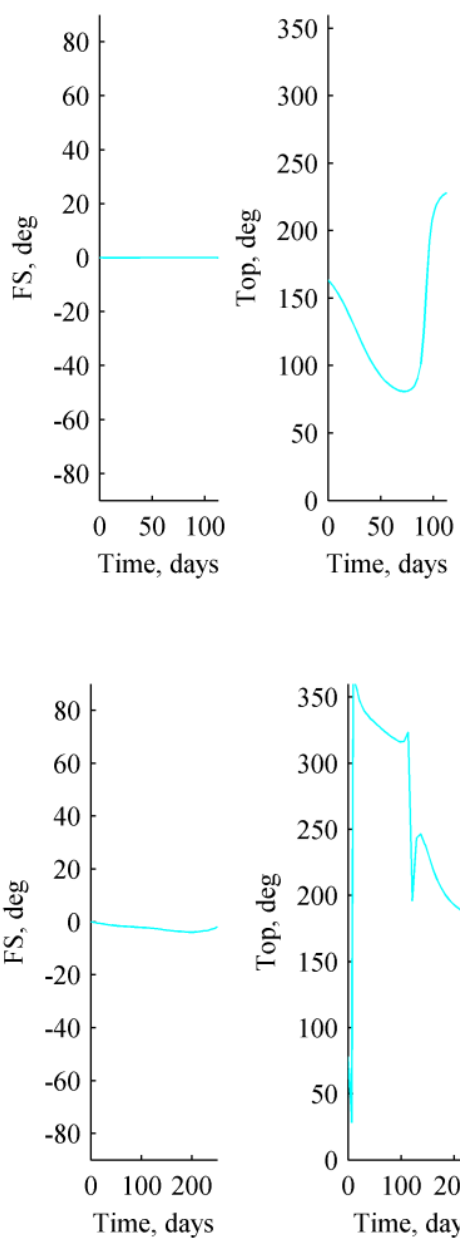

b)

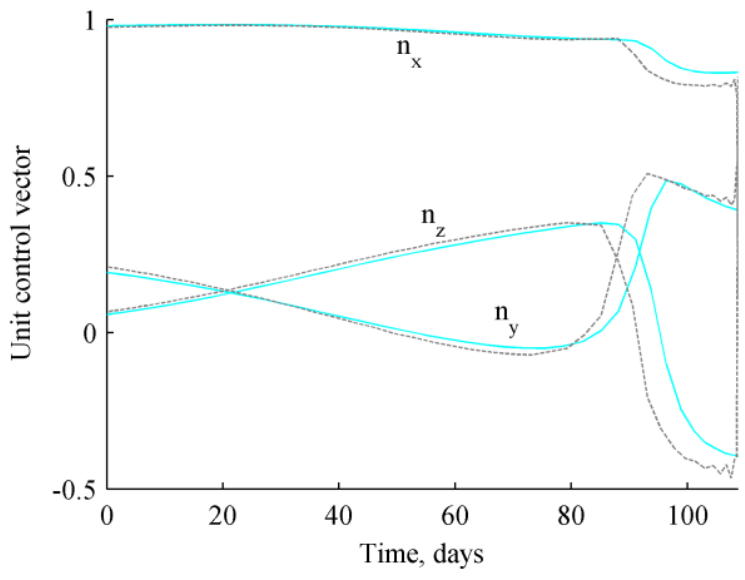

d)

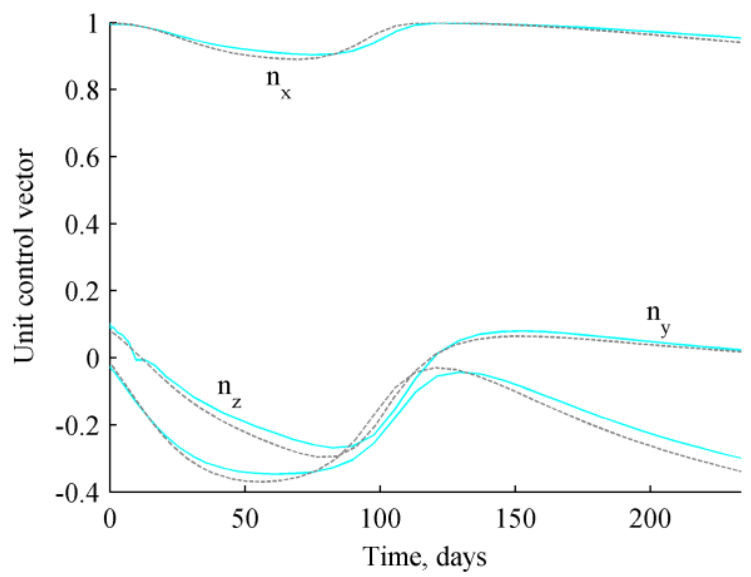

Figure 15 Control profiles of time-optimal extended mission concepts. Dashed grey lines are for the ideal

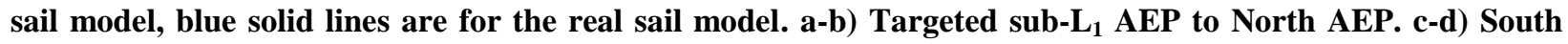
AEP to Parker Spiral AEP.

\section{Conclusions}

This paper has provided a preliminary mission analysis for NASA's Sunjammer solar sail mission, including the selection of accessible artificial equilibrium points (AEPs), solar sail Halo orbits around the true sub- $\mathrm{L}_{1}$ point, optimal transfers from GTO to the sub- $\mathrm{L}_{1}$ region and finally a range of potentially interesting extended mission concepts. From the targeted sub- $\mathrm{L}_{1}$ AEP or solar sail Halo orbit established in this paper, Sunjammer can increase the warning time for space weather events by a factor 1.6 compared to existing infrastructure at the classical $\mathrm{L}_{1}$ point.

All transfers have been designed for both an ideal sail performance and Sunjammer's real performance, where it was shown that the real solar sail data can be accurately represented with a $9^{\text {th }}$ order polynomial fit, allowing a quick computation of the sail performance for any sail attitude. 
Targeting either a sub- $\mathrm{L}_{1}$ AEP or a solar sail Halo orbit (both of which lie around a 5 deg solar exclusion zone to ensure undisturbed communications with Earth), the optimal fly-out from a Falcon 9 midnight GTO takes 191 or 132 days, respectively, for an ideal sail model. The real Sunjammer sail performance introduces a penalty of $7.9 \%$ on this time of flight. Furthermore, for either sail model, the upper stage of the Falcon 9 has to launch the spacecraft from GTO's perigee to almost escape conditions.

The extended mission concepts consider a tour along a set of AEPs to underpin a range of solar sail applications. These include hovering above/below the ecliptic for high-latitude observation of the Earth, hovering ahead of the Earth in the Parker Spiral as well as enabling astronomical observations from the vicinity of the $\mathrm{L}_{2}$ point. In particular, the AEPs visited are: sub- $\mathrm{L}_{1}$ AEP $\rightarrow$ AEP north of the ecliptic $\rightarrow$ AEP south of the ecliptic $\rightarrow$ AEP ahead of the Earth in the Parker Spiral $\rightarrow$ AEP associated to the $\mathrm{L}_{2}$-region. Each leg of the tour has been optimized for the time of flight, resulting in transfer times of between 3 and 8 months. Non-ideal sail properties as taken into account within the real sail model impose an average penalty of $2.4-7.3 \%$ on these transfer times and a penalty of $5.5 \%$ on the total time of flight.

\section{Aknowledgements}

This work was funded by the European Research Council Advanced Investigator Grant-227571: Visionary Space Systems: Orbital Dynamics at Extremes of Spacecraft Length-Scale.

\section{References}

${ }^{1}$ Y. Tsuda, O. Mori, R. Funase, H. Sawada, T. Yamamoto, T. Saiki, T. Endo, J. Kawaguchi, Flight Status of IKAROS Deep Space Solar Sail Demonstrator, Acta Astronautica, 69 (2011) 833-840.

${ }^{2}$ L. Johnson, M. Whorton, A. Heaton, R. Pinson, G. Laue, C. Adams, NanoSail-D: A Solar Sail Demonstration Mission, Acta Astronautica, 68 (2011) 571-575.

${ }^{3}$ C.R. McInnes, A.J. McDonald, J.F.L. Simmons, E.W. MacDonald, Solar Sail Parking in Restricted Three-Body Systems, Journal of Guidance, Control, and Dynamics, 17 (1994) 399-406.

${ }^{4}$ T.J. Waters, C.R. McInnes, Periodic Orbits Above the Ecliptic in the Solar-Sail Restricted Three-Body Problem, Journal of Guidance, Control, and Dynamics, 30 (2007) 687-693.

${ }^{5}$ J. Heiligers, M. Ceriotti, C.R. McInnes, J.D. Biggs, Design of Optimal Transfers Between North and South Pole-Sitter Orbits, in: 22nd AAS/AIAA Space Flight Mechanics Meeting, Charleston, SC, 2012.

${ }^{6}$ J. Heiligers, C.R. McInnes, Agile Solar Sailing in Three-body Problem: Motion Between Artificial Equilibrium Points (IAC-13C1.8.3), in: 64th International Astronautical Congress, Beijing, China, 2013.

7 C.R. McInnes, Solar Sailing: Technology, Dynamics and Mission Applications, Springer-Praxis Books in Astronautical Engineering, Springer-Verlag, Berlin, 1999.

${ }^{8}$ B. Dachwald, G. Mengali, A.A. Quarta, M. Macdonald, Parametric Model and Optimal Control of Solar Sails with Optical Degradation, Journal of Guidance, Control, and Dynamics, 29 (2006) 1170-1178.

9 B. Derbes, D. Lichodziejewski, J. Ellis, D. Scheeres, Sailcraft Coordinate Systems and Format for Reporting Propulsive Performance in: 14th AAS/AIAA Space Flight Mechanics Conference, Maui, Hawaii, 2004.

${ }^{10}$ Goddard Space Flight Center, Advanced Composition Explorer ACE - Detailed Mission Requirements (Report number GSFC410-ACE-017), in, 1995.

${ }^{11}$ H. Baoyin, C. McInnes, Solar Sail Halo Orbits at the Sun-Earth Artificial L1-point, Celestial Mechanics and Dynamical Astronomy, 94 (2006) 155-171.

${ }^{12}$ K.C. Howell, Three-Dimensional, Periodic, 'Halo' Orbits, Celestial Mechanics and Dynamical Astronomy, 32 (1983) 53-71.

${ }^{13}$ V.M. Becerra, Solving Complex Optimal Control Problems at No Cost with PSOPT, in: IEEE Multi-conference on Systems and Control, Yokohama, Japan, 2010.

${ }^{14}$ J. Heiligers, M. Ceriotti, C.R. McInnes, J.D. Biggs, Mission Analysis and Systems Design of a Near-Term and Far-Term PoleSitter Mission, Acta Astronautica, 94 (2014). 
${ }^{15}$ M. Ceriotti, J. Heiligers, C.R. McInnes, Trajectory and Spacecraft Design for a Pole-Sitter Mission, Journal of Spacecraft and Rockets, (2013, In Press).

${ }^{16}$ S. Milan, KuaFu: Exploring the Sun-Earth Connection, Astronomy \& Geophysics, 53 (2012) 4.21-24.24.

${ }^{17}$ J. Heiligers, M. Ceriotti, C.R. McInnes, J.D. Biggs, Design of optimal Earth pole-sitter transfers using low-thrust propulsion Acta Astronautica, 79 (2012) 253-268 